TRANSACTIONS OF THE

AMERICAN MATHEMATICAL SOCIETY

Volume 360, Number 11, November 2008, Pages 5805-5830

S 0002-9947(08)04415-2

Article electronically published on June 19, 2008

\title{
LOW REGULARITY SOLUTIONS FOR A 2D QUADRATIC NONLINEAR SCHRÖDINGER EQUATION
}

\author{
IOAN BEJENARU AND DANIELA DE SILVA
}

\begin{abstract}
We establish that the initial value problem for the quadratic nonlinear Schrödinger equation

$$
i u_{t}-\Delta u=u^{2}
$$

where $u: \mathbb{R}^{2} \times \mathbb{R} \rightarrow \mathbb{C}$, is locally well-posed in $H^{s}\left(\mathbb{R}^{2}\right)$ when $s>-1$. The critical exponent for this problem is $s_{c}=-1$, and previous work by Colliander, Delort, Kenig and Staffilani, 2001, established local well-posedness for $s>$ $-3 / 4$.
\end{abstract}

\section{INTRODUCTION}

The aim of this paper is to continue the development of the theory for quadratic nonlinear Schrödinger equations of the form:

$$
\left\{\begin{aligned}
i u_{t}-\Delta u & =P(u, \bar{u}), t \in \mathbb{R}, x \in \mathbb{R}^{n}, \\
u(x, 0) & =u_{0}(x) \in H^{s}\left(\mathbb{R}^{n}\right),
\end{aligned}\right.
$$

where $u: \mathbb{R}^{n} \times \mathbb{R} \rightarrow \mathbb{C}$ and $P: \mathbb{C}^{2} \rightarrow \mathbb{C}$ is a quadratic polynomial. Here $H^{s}\left(\mathbb{R}^{n}\right)$ denotes the usual inhomogeneous Sobolev space.

An important concept for this type of problem is the scaling (critical) exponent $s_{c}$. This is the exponent of the Sobolev space which scales in the same way as the equation. A straightforward computation shows that the critical exponent for problem (1.1) is $s_{c}=\frac{n}{2}-2$. Heuristically one would expect to obtain a local wellposedness theory for initial data $u_{0} \in H^{s}$ for all $s \geq s_{c}$. For a precise definition of local well-posedness, we refer to the statement of our main result, Theorem 1.1 .

As for many other evolution equations of this type, it turns out that in lower dimensions obtaining local well-posedness for $s \geq s_{c}$ is a delicate problem. Indeed, for $n \geq 4$ it has been shown in [5], via Strichartz estimates, that local well-posedness holds for (1.1) for all $s \geq s_{c}$, while for $n \leq 3$ local well-posedness holds for all $s \geq 0$. On the other hand, for $n \leq 3, s_{c}=\frac{n}{2}-2<0$, it is therefore expected that one needs more refined techniques in order to get closer to the scaling exponent.

One new feature in low dimension is that one has to distinguish among the three types of nonlinearities $u^{2},|u|^{2}=u \bar{u}$ and $\bar{u}^{2}$. These nonlinearities behave differently, and the local well-posedness results obtained for each of them are not the same.

Received by the editors August 21, 2006.

2000 Mathematics Subject Classification. Primary 35Q55.

The authors were partially supported by the Mathematical Sciences Research Institute (MSRI) at Berkeley. 
In the case $n=3\left(s_{c}=-\frac{1}{2}\right)$ [13] established local well-posedness for $s>-\frac{1}{2}$ for the nonlinearities $u^{2}$ and $\bar{u}^{2}$, while local well-posedness for the nonlinearity $|u|^{2}$ is shown to hold for $s>-\frac{1}{4}$. The open problem in $3 D$ is what happens at scaling, i.e. $s=-\frac{1}{2}$ for $u^{2}$ and $\bar{u}^{2}$ and $s=-\frac{1}{4}$ for $|u|^{2}$. We have strong reasons to believe that for the nonlinearity $|u|^{2}$ one cannot get a positive result for $s<-\frac{1}{4}$.

In dimensions one and two, the available results are even further away from scaling. Precisely, in one dimension $\left(s_{c}=-\frac{3}{2}\right)$ the following results are known:

- for $u^{2}$ : well-posedness for $s \geq-1$ and ill-posedness for $s<-1$; see [1;

- for $|u|^{2}$ : well-posedness for $s>-\frac{1}{4}$; see [8];

- for $\bar{u}^{2}$ : well-posedness for $s>-\frac{3}{4}$; see [8].

We remark that the technique in 1 is not directly applicable to improving the results known for the nonlinearities $|u|^{2}$ and $\bar{u}^{2}$. Indeed such a technique is based on "undoing" the counterexample in 8 imposing the condition $s>-3 / 4$ for the nonlinearity $u^{2}$. For $|u|^{2}$, the authors of [1] could not undo the counterexample yielding $s>-\frac{1}{4}$, while the nonlinearity $\bar{u}^{2}$ is known as being the easiest of all, and a simpler argument should suffice. Hence, work is needed for these two cases.

For $n=2$ the state of art was established in 6]. For the nonlinearities $u^{2}$ and $\bar{u}^{2}$ the authors proved well-posedness for $s>-\frac{3}{4}$, while for the nonlinearity $|u|^{2}$ well-posedness is shown to hold for $s>-\frac{1}{4}$.

Before the result in [1], the breakpoints for $u^{2}$ in $1 D$ and $2 D$, namely $s>$ $-\frac{3}{4}$, were imposed by a very similar counterexample; see [8] and [6] respectively. Therefore we decided to investigate a possible improvement for the nonlinearity $u^{2}$ in $2 D$ following the ideas in 1 . We have used similar structures for the functional spaces introduced to overcome the deadlock imposed by the known counterexample in 6. The techniques are more involved since we deal with the two-dimensional problem which brings additional difficulties.

Recall that we are interested in the problem

$$
\left\{\begin{array}{c}
i u_{t}-\Delta u=u^{2}, t \in \mathbb{R}, x \in \mathbb{R}^{2}, \\
u(x, 0)=u_{0}(x) \in H^{s}\left(\mathbb{R}^{2}\right) .
\end{array}\right.
$$

We prove the following result.

Theorem 1.1. Let $s>-1$ and $r>0$ be any radius, and let $B_{r}$ be the ball

$$
B_{r}:=B_{H_{x}^{s}(\mathbb{R})}(0, r):=\left\{u_{0} \in H_{x}^{s}(\mathbb{R}):\left\|u_{0}\right\|_{H_{x}^{s}(\mathbb{R})}<r\right\} .
$$

Then there exists a time $T>0$ and a map $f \mapsto u[f]$ which is continuous from $B_{r}$ to $C_{t}^{0} H_{x}^{s}([0, T] \times \mathbb{R})$, such that the restriction of this map to $B_{r} \cap H_{x}^{s^{\prime}}(\mathbb{R})$ (with the $H_{x}^{s^{\prime}}(\mathbb{R})$ topology) maps continuously to $C_{t}^{0} H^{s^{\prime}}([0, T] \times \mathbb{R})$ for any $s^{\prime} \geq$ s. Furthermore, if $f$ lies in a smooth space, say $B_{r} \cap H_{x}^{3}(\mathbb{R})$, then $u[f]$ lies in $C_{t}^{0} H_{x}^{3} \cap C_{t}^{1} H_{x}^{1}([0, T] \times \mathbb{R})$ and solves equation (1.2) in the classical sense.

Our result does not say or predict anything about the behavior of (1.2) at scaling, i.e. for $u_{0} \in H^{-1}$. Potentially we could prove some logarithmic divergence in the bilinear estimate in the spirit of [11. However this would not say much about the well/ill-posedness of the problem in this case.

Concerning the nonlinearities $|u|^{2}$ and $\bar{u}^{2}$ we believe that our paper together with 1 provide most of the tools needed for expanding the known results both in the $1 D$ and $2 D$ cases. This would establish a satisfactory well-posedness theory in $1 D$ and $2 D$ for (1.1). 
The formalism needed for our result was developed in [1]. In the next section, Section 2, we provide a concise but rigorous definition of the functional space in which we need to work. For more details about the motivation for the particular structure of such a function space, we refer the reader to [1. Sections 3 through 6 are devoted to the main estimates of the paper, namely the bilinear estimates in the functional space introduced in Section 2.

\section{Description of the FUnCtion SPACE $W^{s}$}

In this section we introduce some notation and we define the function space $W^{s}$ whose properties will allow us to develop a local well-posedness theory for the initial value problem (1.2), for all $s>-1$. We fix $T=1$ and build our space adapted to this scale. A standard rescaling argument gives us the result for all $T$ 's.

Throughout this paper we use the notation $A \lesssim B$ to mean that $A \leq C B$ for some $C$ which may change from line to line, but which is independent of any of the possible variables in our problem. If $A \lesssim B$ and $B \lesssim A$ we say that $A \approx B$. In addition $\langle a\rangle:=1+|a|$ and $a+:=a+\epsilon$ with $\epsilon$ positive and small.

For any $s, b \in \mathbb{R}$, we define $\hat{X}^{s, b}$ to be the closure of the smooth functions $f: \mathbb{R}^{2} \times \mathbb{R} \rightarrow \mathbb{C}$ under the following norm:

$$
\|f\|_{\hat{X}^{s, b}}:=\left\|\langle\xi\rangle^{s}\left\langle\tau-|\xi|^{2}\right\rangle^{b} f\right\|_{L_{\xi}^{2} L_{\tau}^{2}}
$$

These are the Fourier transforms of the usual $X^{s, b}$ spaces defined in [4].

The function space $W^{s}$ will be a modification of $\hat{X}^{s, 1 / 2+}$. As remarked in the Introduction, a close analysis of [6] reveals that in order to obtain well-posedness below $s=-3 / 4$ one has to deal with the same kind of "bad interactions" which appear in the 1-dimensional case. For this reason we follow [1] closely towards the construction of the space $W^{s}$.

Since $W^{s}$ will be constructed directly on the Fourier side (like $\hat{X}^{s, b}$ ), we require that $W^{s}$ satisfies the following properties:

- Monotonicity: If $|f| \leq|g|$ pointwise, then $\|f\|_{W^{s}} \leq\|g\|_{W^{s}}$. In particular, $\|f\|_{W^{s}}=\||f|\|_{W^{s}}$.

- $H^{s}$ Energy estimate:

$$
\left\|\langle\xi\rangle^{s} f\right\|_{L_{\xi}^{2} L_{\tau}^{1}} \lesssim\|f\|_{W^{s}}
$$

- Homogeneous $H^{s}$ solution estimate:

$$
\|f\|_{W^{s}} \lesssim\|f\|_{\hat{X}^{s, 100}} .
$$

- Bilinear estimate:

$$
\left\|\left\langle\tau-|\xi|^{2}\right\rangle^{-1} f * g\right\|_{W^{s}} \lesssim\|f\|_{W^{s}}\|g\|_{W^{s}},
$$

where $f * g$ denotes space-time convolution

$$
f * g(\xi, \tau):=\int_{\mathbb{R}} \int_{\mathbb{R}^{2}} f\left(\xi_{1}, \tau_{1}\right) g\left(\xi_{2}, \tau_{2}\right) d \xi_{1} d \tau_{1}
$$

using the convention

$$
\left(\xi_{1}, \tau_{1}\right)+\left(\xi_{2}, \tau_{2}\right)=(\xi, \tau) .
$$

Once we determine a function space $W^{s}$ that satisfies all of the properties above, the machinery developed in [1] will give the result in Theorem 1.1. 
This section provides the construction of $W^{s}$ and the (almost) trivial check that $W^{s}$ satisfies the first three properties above. The next sections will deal with the bilinear estimate (2.3), which is indeed the core of our paper.

In order to define $W^{s}$, we shall partition the frequency space $(\xi, \tau)$ in the sets $A_{j} \cap B_{d}$ for $j, d \geq 0$, where the $A_{j}$ 's are the annuli

$$
A_{j}:=\left\{(\xi, \tau) \in \mathbb{R} \times \mathbb{R}^{2}: 2^{j} \leq\langle\xi\rangle<2^{j+1}\right\},
$$

while the $B_{d}$ 's are the parabolic neighborhoods

$$
B_{d}:=\left\{(\xi, \tau) \in \mathbb{R} \times \mathbb{R}^{2}: 2^{d} \leq\left\langle\tau-|\xi|^{2}\right\rangle<2^{d+1}\right\} .
$$

In the next sections, we will also use the annuli $C_{m}$ 's, $m \geq 0$, defined by

$$
C_{m}:=\left\{(\xi, \tau) \in \mathbb{R} \times \mathbb{R}^{2}: 2^{m} \leq\langle\tau\rangle<2^{m+1}\right\} .
$$

Moreover, we denote

$$
A_{\leq j}:=\bigcup_{j^{\prime} \leq j} A_{j^{\prime}}, \quad B_{\leq d}:=\bigcup_{d^{\prime} \leq d} B_{d^{\prime}},
$$

and similarly one can define $A_{\geq j}, A_{>j}, B_{\geq d}, B_{>d}$, etc. Finally for any smooth $f$, we denote

$$
\begin{gathered}
f_{j}:=\chi_{A_{j}} f, \\
f_{j, d}:=\chi_{A_{j} \cap B_{d}} f,
\end{gathered}
$$

where $\chi_{A}$ is the characteristic function of a set $A$. We then have 1

$$
\|f\|_{\hat{X}^{s, b}} \approx\left(\sum_{j} \sum_{d} 2^{2 s j} 2^{2 b d}\left\|f_{j, d}\right\|_{L_{\xi}^{2} L_{\tau}^{2}}^{2}\right)^{1 / 2}
$$

Now define the space $\hat{X}^{s, b, 1}$, that is, the Besov endpoint refinement of $\hat{X}^{s, b}$, by the following norm:

$$
\|f\|_{\hat{X}^{s, b, 1}}:=\left(\sum_{j} 2^{2 s j}\left(\sum_{d} 2^{b d}\left\|f_{j, d}\right\|_{L_{\xi}^{2} L_{\tau}^{2}}\right)^{2}\right)^{1 / 2} .
$$

If $b=\frac{1}{2}$, a straightforward computation shows that $\hat{X}^{s, \frac{1}{2}} \subset \hat{X}^{s, \frac{1}{2}, 1} \subset \hat{X}^{s, \frac{1}{2}+}$. This Besov-type space allows us to handle the "parallel interaction" case, that is, the case when the nonlinearity interacts two components of the solution $u$ with the same high frequency. However, this space alone is not sufficient to handle even the endpoint $s=-3 / 4$, because of a divergence near the " $\tau$ " axis. Thus we need to further modify this space toward the definition of $W^{s}$. We introduce the following function space $Y^{s}$, defined via the norm

$$
\begin{aligned}
\|f\|_{Y^{s}} & :=\left\|\langle\xi\rangle^{s} f\right\|_{L_{\xi}^{2} L_{\tau}^{1}}+\left\|\langle(\xi, \tau)\rangle^{s+1} f\right\|_{L_{\xi}^{2} L_{\tau}^{2}} \\
& :=\|f\|_{\langle\xi\rangle^{s} L_{\xi}^{2} L_{\tau}^{1}}+\|f\|_{\langle(\xi, \tau)\rangle^{s+1} L_{\xi}^{2} L_{\tau}^{2}}
\end{aligned}
$$

which we denote by $|(\xi, \tau)|=\left(|\tau|+|\xi|^{2}\right)^{\frac{1}{2}}$. Then we define the sum

$$
Z^{s}:=\hat{X}^{s, 1 / 2,1}+Y^{s}
$$

which is endowed with the usual norm

$$
\|f\|_{Z^{s}}:=\inf \left\{\left\|f_{1}\right\|_{\hat{X}^{s, 1 / 2,1}}+\left\|f_{2}\right\|_{Y^{s}}: f_{1} \in \hat{X}^{s, 1 / 2,1} ; f_{2} \in Y^{s} ; f=f_{1}+f_{2}\right\} .
$$

\footnotetext{
${ }^{1}$ All sums and unions involving $j$ and $d$ shall be over the nonnegative integers unless otherwise mentioned.
} 
It is easy to verify that $Z^{s}$ is a Banach space, with the Schwartz functions being dense. Using this space we will be able to handle the divergences occurring along the time axis. By definition,

$$
\|f\|_{Z^{s}} \leq\|f\|_{\hat{X}^{s, 1 / 2,1}},\|f\|_{Y^{s}} .
$$

Vice-versa, in order to prove a linear estimate of the form $\|T f\|_{Z^{s}} \lesssim\|f\|_{Z^{s}}$, it suffices to prove both the estimates $\|T f\|_{Z^{s}} \lesssim\|f\|_{\hat{X}^{s, 1 / 2,1}}$, and $\|T f\|_{Z^{s}} \lesssim\|f\|_{Y^{s}}$.

It is a simple exercise to establish that if $f$ is smooth, then

$$
\|f\|_{\langle\xi\rangle^{s} L_{\xi}^{2} L_{\tau}^{1}} \lesssim\|f\|_{Z^{s}} \text { and }\|f\|_{Z^{s}} \lesssim\|f\|_{\hat{X}^{s, 90}} .
$$

We remark that because of the $L^{2}$ structure of the spaces involved in our analysis we have the following localization property:

$$
\|f\|_{\mathcal{X}} \approx\left(\sum_{j}\left\|f_{j}\right\|_{\mathcal{X}}^{2}\right)^{1 / 2}
$$

for $\mathcal{X}$ either of $\hat{X}^{s, 1 / 2,1}, Y^{s}$, or $Z^{s}$.

The two spaces $\hat{X}^{s, 1 / 2,1}$ and $Y^{s}$ paste together nicely along the fuzzy boundary $\left\langle\tau-|\xi|^{2}\right\rangle \approx\langle\xi\rangle^{2}$. More precisely, the following lemma holds (for details of the proof see [1]).

Lemma 2.1 (Pasting lemma). Let $f$ be a smooth function and let $-1 \leq s<0$. If $f$ is supported on $\bigcup_{j} A_{j} \cap B_{\geq 2 j-100}$, then

$$
\|f\|_{Y^{s}} \lesssim\|f\|_{Z^{s}}
$$

Conversely, if $f$ is supported on $\bigcup_{j} A_{j} \cap B_{\leq 2 j+100}$, then

$$
\|f\|_{\hat{X}^{s, 1 / 2,1}} \lesssim\|f\|_{Z^{s}} .
$$

Now, set

$$
K=\bigcup_{j}\left(A_{j} \cap B_{\leq 2 j-4}\right) .
$$

Notice that on $K \cap A_{j}$ we have $2^{2 j-4} \leq \tau \leq 2^{2 j+4}$. For a smooth function $f$, we define

$$
f^{K}:=\chi_{K} f, f^{K^{c}}:=\chi_{K^{c}} f .
$$

Here $K^{c}$ denotes the complement of $K$. Then, the pasting lemma implies that

$$
\|f\|_{Z^{s}} \approx\left\|f^{K}\right\|_{\hat{X}^{s, \frac{1}{2}, 1}}+\left\|f^{K^{c}}\right\|_{Y^{s}}
$$

This is the way we essentially think of measuring functions in $Z^{s}$.

The space $Z^{s}$ is a good candidate for $W^{s}$, as it is able to cope with two of the dangerous quadratic interactions in the equation (namely the parallel interactions, and the interactions which output near the time axis). There is a third type of interaction which could cause trouble: when a solution component near the parabola $\left\{\tau=|\xi|^{2}\right\}$ interacts with a component near the reflected parabola $\left\{\tau=-|\xi|^{2}\right\}$ to create a large contribution near the frequency origin. However, we do expect the solution to stay concentrated in the upper half-plane $\tau>0$. To exploit this fact we shall introduce a weight

$$
w(\xi, \tau):=\max (1,-\tau)^{10}
$$

to localize to the upper half-plane, and define $W^{s}$ to be the space

$$
\|f\|_{W^{s}}:=\|w f\|_{Z^{s}}
$$


The first three properties that $W^{s}$ is required to satisfy (see the beginning of this section) are straightforward. The monotonicity of $\hat{X}^{s, 1 / 2,1}, Y^{s}$ and hence of $W^{s}$ is immediate. The $H^{s}$ energy estimate (2.1) follows from (2.7) (since $w \geq 1$ ), while the homogeneous $H^{s}$ solution estimate (2.2) follows directly from the following inequalities:

$$
\|f\|_{W^{s}} \leq\|w f\|_{\hat{X}^{s, 1 / 2,1}} \lesssim\|w f\|_{\hat{X}^{s, 90}} \lesssim\|f\|_{\hat{X}^{s, 100}}
$$

where we have used the crude estimate $w(\xi, \tau) \leq C\left\langle\tau-|\xi|^{2}\right\rangle^{10}$.

It remains to establish the bilinear estimate (2.3). Applying (2.13) and monotonicity, we reduce to showing that

$$
\left\|\frac{w}{\left\langle\tau-|\xi|^{2}\right\rangle}\left(\frac{f}{w} * \frac{g}{w}\right)\right\|_{Z^{s}} \lesssim\|f\|_{Z^{s}}\|g\|_{Z^{s}}
$$

for all nonnegative smooth functions $f, g$.

Using (2.14) together with the pasting lemma, it follows that (2.3) can be deduced by the bilinear estimates stated in the following proposition.

Proposition 2.2. Let $f, g$ be nonnegative smooth functions and let $-1<s<0$. Then,

$$
\begin{gathered}
\left\|\frac{w}{\left\langle\tau-|\xi|^{2}\right\rangle}\left(\frac{f^{K^{c}}}{w} * \frac{g^{K^{c}}}{w}\right)\right\|_{Z^{s}} \lesssim\left\|f^{K^{c}}\right\|_{Y^{s}}\left\|g^{K^{c}}\right\|_{Y^{s}} \\
\left\|\frac{w}{\left\langle\tau-|\xi|^{2}\right\rangle}\left(\frac{f^{K}}{w} * \frac{g^{K}}{w}\right)\right\|_{Z^{s}} \lesssim\left\|f^{K}\right\|_{\hat{X}^{s, \frac{1}{2}, 1}}\left\|g^{K}\right\|_{\hat{X}^{s, \frac{1}{2}, 1}} \\
\left\|\frac{\chi_{K} w}{\left\langle\tau-|\xi|^{2}\right\rangle}\left(\frac{f^{K}}{w} * \frac{g^{K^{c}}}{w}\right)\right\|_{\hat{X}^{s, \frac{1}{2}, 1}} \lesssim\left\|f^{K}\right\|_{\hat{X}^{s, \frac{1}{2}, 1}}\left\|g^{K^{c}}\right\|_{Y^{s}} \\
\left\|\frac{\left(1-\chi_{K}\right) w}{\left\langle\tau-|\xi|^{2}\right\rangle}\left(\frac{f^{K}}{w} * \frac{g^{K^{c}}}{w}\right)\right\|_{Y^{s}} \lesssim\left\|f^{K}\right\|_{\hat{X}^{s, \frac{1}{2}, 1}}\left\|g^{K^{c}}\right\|_{Y^{s}} .
\end{gathered}
$$

Remark 2.3. For $\tau=\tau_{1}+\tau_{2}, \xi=\xi_{1}+\xi_{2}$ we have

$$
w(\xi, \tau) \leq C w\left(\xi_{1}, \tau_{1}\right) w\left(\xi_{2}, \tau_{2}\right)
$$

hence we get the following pointwise estimate:

$$
\frac{w}{\left\langle\tau-|\xi|^{2}\right\rangle}\left(\frac{f}{w} * \frac{g}{w}\right) \leq \frac{C}{\left\langle\tau-|\xi|^{2}\right\rangle}(f * g),
$$

which will turn out to be quite useful in the proof of Proposition 2.2.

\section{The proof of the estimate (2.15)}

In this section we present the proof of the bilinear estimate (2.15). We start with a simple auxiliary lemma. Here and henceforth, we will abbreviate $L_{\xi}^{2} L_{\tau}^{2}$ by $L^{2}$. Analogously we denote by $L^{\infty}$ the mixed Lebesgue space $L_{\xi}^{\infty} L_{\tau}^{\infty}$.

Lemma 3.1. Let $f, g$ be nonnegative smooth functions. Then

$$
\begin{aligned}
& \left\|\frac{1-\chi_{K}}{\left\langle\tau-|\xi|^{2}\right\rangle}(f * g)_{k, d}\right\|_{L^{2}} \lesssim 2^{-d}\left(2^{k+\frac{d}{2}}\right)\|f\|_{L^{2}}\|g\|_{L^{2}} \\
& \left\|\left(f * \chi_{C_{n}} g\right)_{k, d}\right\|_{L_{\xi}^{2} L_{\tau}^{1}} \lesssim 2^{k+\frac{n+d}{2}}\|f\|_{L^{2}}\left\|\chi_{C_{n}} g\right\|_{L^{2}} .
\end{aligned}
$$


Proof. Let us prove (3.1). We have

$$
\begin{aligned}
& \left\|\frac{1-\chi_{K}}{\left\langle\tau-|\xi|^{2}\right\rangle}(f * g)_{k, d}\right\|_{L^{2}} \leq\left\|\left(1-\chi_{K}\right) \frac{\chi_{A_{k} \cap B_{d}}}{\left\langle\tau-|\xi|^{2}\right\rangle}\right\|_{L^{2}}\|f * g\|_{L^{\infty}} \\
& \leq\left\|\left(1-\chi_{K}\right) \frac{\chi_{A_{k} \cap B_{d}}}{\left\langle\tau-|\xi|^{2}\right\rangle}\right\|_{L^{2}}\|f\|_{L^{2}}\|g\|_{L^{2}}
\end{aligned}
$$

where in the last step we have used Young's inequality. Moreover,

$$
\left\|\left(1-\chi_{K}\right) \frac{\chi_{A_{k} \cap B_{d}}}{\left\langle\tau-|\xi|^{2}\right\rangle}\right\|_{L^{2}} \lesssim 2^{-d}\left(2^{k+\frac{d}{2}}\right)
$$

since $\left\langle\tau-|\xi|^{2}\right\rangle \approx 2^{-d}$ on $B_{d}$, and the variables $\xi$ and $\tau$ are confined to sets of measure $2^{2 k}$ and $2^{d}$, respectively.

We now prove (3.2). We have

$$
\begin{gathered}
\left\|\left(f * \chi_{C_{n}} g\right)_{k, d}\right\|_{L_{\xi}^{2} L_{\tau}^{1}} \leq\left\|\chi_{A_{k} \cap B_{d}}\right\|_{L^{2}}\left\|f * \chi_{C_{n}} g\right\|_{L^{2}} \\
\lesssim 2^{k+\frac{d}{2}}\|f\|_{L^{2}}\left\|\chi_{C_{n}} g\right\|_{L_{\xi}^{2} L_{\tau}^{1}} \lesssim 2^{k+\frac{d+n}{2}}\|f\|_{L^{2}}\|g\|_{L^{2}}
\end{gathered}
$$

where in obtaining (3.3) we used Cauchy-Schwartz in $\tau$, while in obtaining (3.4) we used the fact that the variables $\xi$ and $\tau$ are confined to sets of measure $2^{2 k}$ and $2^{d}$, respectively, together with Young's inequality. In the last step we have then used Cauchy-Schwartz in $\tau$ and the fact that $\tau$ describes a set of measure approximately $2^{n}$.

We are now ready to prove our bilinear estimate.

Proof of (2.15). We wish to prove that

$$
\left\|\frac{w}{\left\langle\tau-|\xi|^{2}\right\rangle}\left(\frac{f^{K^{c}}}{w} * \frac{g^{K^{c}}}{w}\right)\right\|_{Z^{s}} \lesssim\left\|f^{K^{c}}\right\|_{Y^{s}}\left\|g^{K^{c}}\right\|_{Y^{s}} .
$$

Define $h:=f^{K^{c}} * g^{K^{c}}$. Then using the definition of the space $Y^{s}$, the pasting lemma, and Remark 2.3 , the desired estimate will follow from the bilinear estimates:

$$
\begin{gathered}
\left\|\frac{\chi_{K}}{\left\langle\tau-|\xi|^{2}\right\rangle} h\right\|_{\hat{X}^{s, 1 / 2,1}} \lesssim\left\|f^{K^{c}}\right\|_{Y^{s}}\left\|g^{K^{c}}\right\|_{Y^{s}}, \\
\left\|\frac{1-\chi_{K}}{\left\langle\tau-|\xi|^{2}\right\rangle} h\right\|_{\langle(\xi, \tau)\rangle^{s+1} L^{2}} \lesssim\left\|f^{K^{c}}\right\|_{Y^{s}}\left\|g^{K^{c}}\right\|_{Y^{s}}, \\
\left\|\frac{w}{\left\langle\tau-|\xi|^{2}\right\rangle}\left(1-\chi_{K}\right)\left(\frac{f}{w} * \frac{g}{w}\right)\right\|_{\langle\xi\rangle^{s} L_{\xi}^{2} L_{\tau}^{1}} \lesssim\left\|f^{K^{c}}\right\|_{Y^{s}}\left\|g^{K^{c}}\right\|_{Y^{s}} .
\end{gathered}
$$

We divide our proof into three steps.

Step 1. Proof of (3.5).

We decompose

$$
h_{k}=\sum_{i, j}\left(f_{i}^{K^{c}} * g_{j}^{K^{c}}\right)_{k},
$$

where $h_{k}=h_{k}(\xi, \tau), f_{i}=f_{i}\left(\xi_{1}, \tau_{1}\right)$ and $g_{j}=g_{j}\left(\xi_{2}, \tau_{2}\right)$ with $(\xi, \tau)=\left(\xi_{1}, \tau_{1}\right)+$ $\left(\xi_{2}, \tau_{2}\right)$. Hence, in order for $h_{k}$ to be nonzero, we must have that any of the following cases holds:

- $j \leq i-11,|i-k| \leq 2$,

- $i \leq j-11,|j-k| \leq 2$,

- $|i-j| \leq 10, k \leq \max \{i, j\}+1$. 
Assume by symmetry that $i \leq j$. Then,

$$
\begin{aligned}
h_{k} & =\sum_{i \leq j-11 ;|j-k| \leq 2}\left(f_{i}^{K^{c}} * g_{j}^{K^{c}}\right)_{k}+\sum_{k \leq j+1 ; i \leq j \leq i+10}\left(f_{i}^{K^{c}} * g_{j}^{K^{c}}\right)_{k} \\
& =h_{k}^{\prime}+h_{k}^{\prime \prime} .
\end{aligned}
$$

A straightforward computation shows that

$$
\left\|\frac{1}{\left\langle\tau-|\xi|^{2}\right\rangle}\right\|_{L_{\xi}^{2} L_{\tau}^{2}\left(A_{k} \cap B_{d}\right)} \leq 2^{-d / 2+k}
$$

which will be used to analyze both $h_{k}^{\prime}$ and $h_{k}^{\prime \prime}$.

We start by estimating $h_{k}^{\prime}$. By the definition of $\hat{X}^{s, 1 / 2,1}$ and of $K$, we get that $\left\|\frac{\chi_{K} h_{k}^{\prime}}{\left\langle\tau-|\xi|^{2}\right\rangle}\right\|_{\hat{X}^{s, 1 / 2,1}}^{2}$ is bounded by

$$
\begin{aligned}
& 2^{2 k s}\left(\sum_{d \leq 2 k-4} \sum_{i \leq j-11 ;|j-k| \leq 2} 2^{\frac{d}{2}}\left\|\frac{1}{\left\langle\tau-|\xi|^{2}\right\rangle}\left(f_{i}^{K^{c}} * g_{j}^{K^{c}}\right)_{k, d}\right\|_{L^{2}}\right)^{2} \\
& \lesssim\left(\sum_{d \leq 2 k-4} \sum_{i \leq j-11 ;|j-k| \leq 2} 2^{\frac{d}{2}+k s} \sum_{d_{1} \geq 2 i-4 ; d_{2} \geq 2 j-4}\left\|\frac{1}{\left\langle\tau-|\xi|^{2}\right\rangle}\left(f_{i, d_{1}}^{K^{c}} * g_{j, d_{2}}^{K^{c}}\right)_{k, d}\right\|_{L^{2}}\right)^{2} .
\end{aligned}
$$

In order to estimate this last term, we notice that in light of the relation

$$
\tau-|\xi|^{2}=\tau_{1}-\left|\xi_{1}\right|^{2}+\tau_{2}-\left|\xi_{2}\right|^{2}-2 \xi_{1} \cdot \xi_{2},
$$

nontrivial interactions happen only in one of the following cases:

(i) $\left|d-d_{2}\right| \leq 5, d_{1} \leq d+6$,

(ii) $\left|d-d_{1}\right| \leq 5, d_{2} \leq d+6$,

(iii) $d_{1}, d_{2} \geq d+7,\left|d_{1}-d_{2}\right| \leq 2$.

Using (3.9) together with Hölder's inequality, in all such cases we have that the sum above is bounded by

$$
\begin{aligned}
& \left(\sum_{d \leq 2 k-4} \sum_{i \leq j-11 ;|j-k| \leq 2} 2^{\frac{d}{2}} 2^{-\frac{d}{2}+k(s+1)} \sum_{d_{1} \geq 2 i-4} \sum_{d_{2} \geq 2 j-4}\left\|f_{i, d_{1}}^{K^{c}} * g_{j, d_{2}}^{K^{c}}\right\|_{L^{\infty}}\right)^{2} \\
= & \left(\sum_{d \leq 2 k-4} \sum_{i \leq j-11 ;|j-k| \leq 2} 2^{k(s+1)} \sum_{d_{1} \geq 2 i-4} \sum_{d_{2} \geq 2 j-4}\left\|f_{i, d_{1}}^{K^{c}} * g_{j, d_{2}}^{K^{c}}\right\|_{L^{\infty}}\right)^{2}=S_{k} .
\end{aligned}
$$

In order to estimate $S_{k}$, in each of the cases (i), (ii), and (iii) we will use the following estimate, which follows from Young's inequality together with the definition of the space $Y^{s}$ and the fact that $f^{K^{c}}$ and $g^{K^{c}}$ are supported away from the parabola:

$$
\left\|f_{i, d_{1}}^{K^{c}} * g_{j, d_{2}}^{K^{c}}\right\|_{L^{\infty}} \leq 2^{-\left(d_{1}+d_{2}\right) \frac{(s+1)}{2}}\left\|f_{i, d_{1}}^{K^{c}}\right\|_{Y^{s}}\left\|g_{j, d_{2}}^{K^{c}}\right\|_{Y^{s}} .
$$


Cases (i)-(ii). In these cases, the summation over $d$ is finite, independent of $k$. Using (3.10) and the facts that $d_{1} \geq 2 i-4, d_{2} \geq 2 j-4$, and $s+1>0$, we then get

$$
\begin{aligned}
S_{k} & \lesssim\left(\sum_{i \leq j-11 ;|j-k| \leq 2} 2^{(k-j-i)(s+1)}\left\|f_{i}^{K^{c}}\right\|_{Y^{s}}\left\|g_{j}^{K^{c}}\right\|_{Y^{s}}\right)^{2} \\
& \lesssim\left(\sum_{i} 2^{-i(s+1)}\left\|f_{i}^{K^{c}}\right\|_{Y^{s}}\right)^{2} \sum_{|j-k| \leq 2}\left\|g_{j}^{K^{c}}\right\|_{Y^{s}}^{2} \\
& \lesssim\left\|f^{K^{c}}\right\|_{Y^{s}}^{2}\left(\sum_{p=-2}^{p=2}\left\|g_{p+k}^{K^{c}}\right\|_{Y^{s}}^{2}\right)
\end{aligned}
$$

where in the last inequality we used Cauchy-Schwartz inequality, together with the fact that $s+1>0$. Notice that without this assumption, we could not perform the desired summation.

Therefore, summing over all $k$ 's we get

$$
\sum_{k} S_{k} \lesssim\left\|f^{K^{c}}\right\|_{Y^{s}}^{2}\left\|g^{K^{c}}\right\|_{Y^{s}}^{2}
$$

Case (iii). In this case the summation over $d$ is finite but dependent on $k$, while $\left|d_{1}-d_{2}\right| \leq 2$. Hence, using the relations among the indexes together with the fact that $\left\|f_{i, d_{1}}\right\|_{Y^{s}} \leq\left\|f_{i}^{K^{c}}\right\|_{Y^{s}},\left\|g_{j, d_{2}}^{K^{c}}\right\|_{Y^{s}} \leq\left\|g_{j}\right\|_{Y^{s}}$, we get

$$
\begin{aligned}
S_{k} & \lesssim k^{2}\left(\sum_{i \leq j-11 ;|j-k| \leq 2} 2^{k(s+1)} \sum_{d_{2} \geq 2 j-4} 2^{-d_{2}(s+1)}\left\|f_{i}^{K^{c}}\right\|_{Y^{s}}\left\|g_{j}^{K^{c}}\right\|_{Y^{s}}\right)^{2} \\
& \lesssim k^{2}\left(\sum_{i \leq j-11 ;|j-k| \leq 2} \sum_{d_{2} \geq 2 j-4} 2^{\left(k-\frac{d_{2}}{2}\right)(s+1)} 2^{-\frac{i}{2}(s+1)} 2^{-\frac{k}{2}(s+1)}\left\|f_{i}^{K^{c}}\right\|_{Y^{s}}\left\|g_{j}^{K^{c}}\right\|_{Y^{s}}\right)^{2} \\
& \lesssim k^{2} 2^{-k(s+1)}\left\|f^{K^{c}}\right\|_{Y^{s}}^{2}\left\|g^{K^{c}}\right\|_{Y^{s}}^{2} .
\end{aligned}
$$

Hence summing over all $k$ 's, under the assumption $s+1>0$ we get

$$
\sum_{k} S_{k} \lesssim\left\|f^{K^{c}}\right\|_{Y^{s}}^{2}\left\|g^{K^{c}}\right\|_{Y^{s}}^{2}
$$

Combining (3.11) and (3.12), we then obtain

$$
\sum_{k}\left\|\frac{\chi_{K} h_{k}^{\prime}}{\left\langle\tau-|\xi|^{2}\right\rangle}\right\|_{\hat{X}^{s, 1 / 2,1}}^{2} \lesssim\left\|f^{K^{c}}\right\|_{Y^{s}}^{2}\left\|g^{K^{c}}\right\|_{Y^{s}}^{2}
$$

We now deal with $h_{k}^{\prime \prime}$. From Young's inequality we have

$$
\begin{aligned}
\left\|h_{k}^{\prime \prime}\right\|_{L^{\infty}} & \leq \sum_{k \leq j+1 ; i \leq j \leq i+10} 2^{-(i+j)(s+1)}\left\|f_{i}^{K^{c}}\right\|_{\langle\xi\rangle^{s+1} L^{2}}\left\|g_{j}^{K^{c}}\right\|_{\langle\xi\rangle^{s+1} L^{2}} \\
& \leq \sum_{k \leq j+1 ; i \leq j \leq i+10} 2^{-(i+j)(s+1)}\left\|f_{i}^{K^{c}}\right\|_{Y^{s}}\left\|g_{j}^{K^{c}}\right\|_{Y^{s}}
\end{aligned}
$$


Hence, using (3.9) and the definition of $\hat{X}^{s, 1 / 2,1}$ we get

$$
\begin{aligned}
& \left\|\frac{\chi_{K} h_{k}^{\prime \prime}}{\left\langle\tau-|\xi|^{2}\right\rangle}\right\|_{\hat{X}^{s, 1 / 2,1}}^{2} \\
& \leq 2^{2 k s}\left(\sum_{d \leq 2 k-4} \sum_{k \leq j+1 ; i \leq j \leq i+10} 2^{\frac{d}{2}} 2^{-\frac{d}{2}+k} 2^{-(i+j)(s+1)}\left\|f_{i}^{K^{c}}\right\|_{Y^{s}}\left\|g_{j}^{K^{c}}\right\|_{Y^{s}}\right)^{2} \\
& \lesssim\left(\sum_{k \leq j+1 ; i \leq j \leq i+10} k 2^{(k-(i+j))(s+1)}\left\|f_{i}^{K^{c}}\right\|_{Y^{s}}\left\|g_{j}^{K^{c}}\right\|_{Y^{s}}\right)^{2} \\
& \lesssim\left(\sum_{k \leq j+1 ; i \leq j \leq i+10} k^{2} 2^{2(k-(i+j))(s+1)}\right)\left\|f^{K^{c}}\right\|_{Y^{s}}^{2}\left\|g^{K^{c}}\right\|_{Y^{s}}^{2},
\end{aligned}
$$

where in the last inequality we have used Cauchy-Schwartz twice. Since $s+1>0$, we have that

$$
\begin{aligned}
& \sum_{k} \sum_{k \leq j+1 ; i \leq j \leq i+10} k^{2} 2^{2(k-(i+j))(s+1)} \lesssim \sum_{k} \sum_{k \leq j+1 ; i \leq j \leq i+10} k^{2} 2^{-2 i(s+1)} \\
& \lesssim \sum_{k} k^{2} 2^{-k(s+1)} \sum_{i} 2^{-i(s+1)} \lesssim 1 .
\end{aligned}
$$

Hence summing the inequality above over all $k$ 's we obtain

$$
\sum_{k}\left\|\frac{\chi_{K} h_{k}^{\prime \prime}}{\left\langle\tau-|\xi|^{2}\right\rangle}\right\|_{\hat{X}^{s, 1 / 2,1}}^{2} \lesssim\left\|f^{K^{c}}\right\|_{Y^{s}}^{2}\left\|g^{K^{c}}\right\|_{Y^{s}}^{2} .
$$

Combining (3.8), (3.13) and (3.14) we get the desired estimate (3.5).

We remark that similar computations show that

$$
\sum_{k}\left\|\frac{\chi_{K^{\prime}} h_{k}}{\left\langle\tau-|\xi|^{2}\right\rangle}\right\|_{\hat{X}^{s, 1 / 2,1}} \lesssim\left\|f^{K^{c}}\right\|_{Y^{s}}^{2}\left\|g^{K^{c}}\right\|_{Y^{s}}^{2}
$$

where

$$
K^{\prime}=\bigcup_{j}\left(A_{j} \cap B_{\leq 2 j+4}\right) .
$$

The reason is that for each $A_{j}, K^{\prime}$ brings in a few more modulations than $K$, namely those between $2^{2 j-3}$ and $2^{2 j+4}$, and our argument easily tolerates this adjustment.

Hence, thanks to the pasting lemma (Lemma 2.1), in the proof of (3.6) and (3.7), whenever $(\xi, \tau) \in A_{k} \cap B_{d}$, we can restrict ourselves to the values $d \geq 2 k+4$. A straightforward computation shows that in this regime we have $2^{d-1} \leq|\tau| \leq 2^{d+2}$. Thus, in what follows, we can assume

$$
(\xi, \tau) \in A_{k} \cap B_{d} \Rightarrow|\tau| \approx 2^{d} .
$$

Step 2. Proof of (3.6).

We use the following decomposition:

$$
\left(f^{K^{c}} * g^{K^{c}}\right)_{k, d}=\sum_{m, n}\left(\chi_{C_{m}} f^{K^{c}} * \chi_{C_{n}} g^{K^{c}}\right)_{k, d} .
$$


We observe that, under the assumption (3.16), in order for the summands to be nonzero, one of the following cases must hold:

- $n \leq d+5,|m-d| \leq 5$,

- $m \leq d+5,|n-d| \leq 5$,

- $m, n>d+5,|m-n| \leq 3$.

By symmetry we can then assume

$$
\begin{aligned}
\left(f^{K^{c}} * g^{K^{c}}\right)_{k, d} & =\sum_{n \leq d+5 ;|m-d| \leq 5}\left(\chi_{C_{m}} f^{K^{c}} * \chi_{C_{n}} g^{K^{c}}\right)_{k, d} \\
& +\sum_{m, n \geq d+5 ;|m-n| \leq 3}\left(\chi_{C_{m}} f^{K^{c}} * \chi_{C_{n}} g^{K^{c}}\right)_{k, d} \\
& =I_{k, d}+I I_{k, d} .
\end{aligned}
$$

From (3.1) we get

$$
\left\|\frac{1-\chi_{K}}{\left\langle\tau-|\xi|^{2}\right\rangle} I_{k, d}\right\|_{L^{2}}^{2} \leq 2^{-2 d}\left(2^{2 k+d}\right) \sum_{n \leq d+5 ;|m-d| \leq 5}\left\|\chi_{C_{m}} f^{K^{c}}\right\|_{L^{2}}^{2}\left\|\chi_{C_{n}} g^{K^{c}}\right\|_{L^{2}}^{2} .
$$

Since on the complement of $K, d \geq 2 k-4$, we get (indeed we observed that we can restrict ourselves to $d \geq 2 k+4$ )

$$
\begin{aligned}
& \left\|\frac{1-\chi_{K}}{\left\langle\tau-|\xi|^{2}\right\rangle} I_{k, d}\right\|_{\langle(\xi, \tau)\rangle^{s+1} L^{2}}^{2} \\
& \lesssim 2^{2 k-d} \sum_{n \leq d+5 ;|m-d| \leq 5} 2^{(d-(m+n))(s+1)}\left\|\chi_{C_{m}} f^{K^{c}}\right\|_{\langle\tau\rangle}^{2}{ }^{\frac{s+1}{2} L^{2}}\left\|\chi_{C_{n}} g^{K^{c}}\right\|_{\langle\tau\rangle^{\frac{s+1}{2}} L^{2}}^{2} \\
& \lesssim 2^{2 k-d} \sum_{n \leq d+5 ;|m-d| \leq 5}^{(d-(m+n))(s+1)}\left\|\chi_{C_{m}} f^{K^{c}}\right\|_{Y^{s}}^{2}\left\|\chi_{C_{n}} g^{K^{c}}\right\|_{Y^{s}}^{2}
\end{aligned}
$$

where in the last inequality we used the fact that $s+1>0$. Therefore,

$$
\begin{aligned}
& \sum_{d, k}\left\|\frac{1-\chi_{K}}{\left\langle\tau-|\xi|^{2}\right\rangle} I_{k, d}\right\|_{\langle(\xi, \tau)\rangle^{s+1} L^{2}}^{2} \\
& \lesssim \sum_{d}\left(\sum_{k \leq d / 2+2} 2^{2 k-d}\right) \sum_{n \leq d+5 ;|m-d| \leq 5} 2^{(d-(m+n))(s+1)}\left\|\chi_{C_{m}} f^{K^{c}}\right\|_{Y^{s}}^{2}\left\|\chi_{C_{n}} g^{K^{c}}\right\|_{Y^{s}}^{2} \\
& \lesssim\left\|f^{K^{c}}\right\|_{Y^{s}}^{2}\left\|g^{K^{c}}\right\|_{Y^{s}}^{2} .
\end{aligned}
$$

In order to justify the last inequality, we can simplify the sum in $m$ by taking $m=d$. Then we get

$$
\begin{aligned}
& \sum_{d} \sum_{n \leq d+5} 2^{-n(s+1)}\left\|_{\chi_{C_{d}}} f^{K^{c}}\right\|_{Y^{s}}^{2}\left\|\chi_{C_{n}} g^{K^{c}}\right\|_{Y^{s}}^{2} \\
& \leq\left(\sum_{d}\left\|\chi_{C_{d}} f^{K^{c}}\right\|_{Y^{s}}^{2}\right)\left(\sum_{n} 2^{-n(s+1)}\right)\left\|g^{K^{c}}\right\|_{Y^{s}}^{2} \\
& \lesssim\left\|f^{K^{c}}\right\|_{Y^{s}}^{2}\left\|g^{K^{c}}\right\|_{Y^{s}}^{2}
\end{aligned}
$$

where in the last step we use the assumption $s+1>0$. We can therefore conclude that

$$
\left\|\sum_{d, k} \frac{1-\chi_{K}}{\left\langle\tau-|\xi|^{2}\right\rangle} I_{k, d}\right\|_{\langle(\xi, \tau)\rangle^{s+1} L^{2}} \lesssim\left\|f^{K^{c}}\right\|_{Y^{s}}\left\|g^{K^{c}}\right\|_{Y^{s}}
$$


Analogously, using (3.1) we get

$$
\begin{aligned}
& \sum_{k, d}\left\|\frac{1-\chi_{K}}{\left\langle\tau-|\xi|^{2}\right\rangle} I I_{k, d}\right\|_{\langle(\xi, \tau)\rangle^{s+1} L^{2}} \\
& \lesssim \sum_{d} \sum_{m, n>d+5 ;|m-n| \leq 3} 2^{(d-(m+n)) \frac{(s+1)}{2}}\left\|\chi_{C_{m}} f^{K^{c}}\right\|_{Y^{s}}\left\|\chi_{C_{n}} g^{K^{c}}\right\|_{Y^{s}} \\
& \leq\left(\sum_{d} \sum_{m, n>d+5 ;|m-n| \leq 3} 2^{(d-(m+n)) \frac{(s+1)}{2}}\right)\left\|f^{K^{c}}\right\|_{Y^{s}}\left\|g^{K^{c}}\right\|_{Y^{s}} \\
& \lesssim\left\|f^{K^{c}}\right\|_{Y^{s}}\left\|g^{K^{c}}\right\|_{Y^{s}},
\end{aligned}
$$

where in the last inequality we again used the fact that $s+1>0$. Combining the inequality above with (3.19) and (3.18) we obtain the desired estimate.

Step 3. Proof of (3.7).

Again, we use the decomposition (3.8). We have the following estimate:

$$
\left\|f_{i}^{K^{c}} * g_{j}^{K^{c}}\right\|_{L_{\xi}^{2} L_{\tau}^{1}} \leq\left\|f_{i}^{K^{c}}\right\|_{L_{\xi}^{2} L_{\tau}^{1}}\left\|g_{j}^{K^{c}}\right\|_{L^{1}} \leq 2^{j}\left\|f_{i}^{K^{c}}\right\|_{L_{\xi}^{2} L_{\tau}^{1}}\left\|g_{j}^{K^{c}}\right\|_{L_{\xi}^{2} L_{\tau}^{1}} .
$$

Hence, since in the support of $\left(1-\chi_{K}\right) h_{k},\left.|\tau-| \xi\right|^{2} \mid \gtrsim 2^{2 k}$, we get

$$
\begin{aligned}
& \left\|\frac{1-\chi_{K}}{\left\langle\tau-|\xi|^{2}\right\rangle} h_{k}^{\prime}\right\|_{\langle\xi\rangle^{s} L_{\xi}^{2} L_{\tau}^{1}} \\
& \leq \sum_{j \leq k+5 ;|k-i| \leq 5} 2^{-2 k} 2^{j} 2^{(k-j-i) s}\left\|f_{i}^{K^{c}}\right\|_{\langle\xi\rangle^{s} L_{\xi}^{2} L_{\tau}^{1}}\left\|g_{j}^{K^{c}}\right\|_{\langle\xi\rangle^{s} L_{\xi}^{2} L_{\tau}^{1}} .
\end{aligned}
$$

Therefore, square-summing in $k$ we get

$$
\sum_{k}\left\|\frac{1-\chi_{K}}{\left\langle\tau-|\xi|^{2}\right\rangle} h_{k}^{\prime}\right\|_{\langle\xi\rangle^{s} L_{\xi}^{2} L_{\tau}^{1}}^{2} \lesssim\left\|f^{K^{c}}\right\|_{Y^{s}}^{2}\left\|g^{K^{c}}\right\|_{Y^{s}}^{2}
$$

In order to justify such an inequality, let us simplify the sum in $i$ by taking $i=k$. Then, first using Cauchy-Schwartz and then the fact that $s+1>0$, we obtain

$$
\begin{aligned}
& \sum_{k}\left(\sum_{j \leq k+5} 2^{-2 k+j(1-s)}\left\|f_{j}^{K^{c}}\right\|_{\langle\xi\rangle^{s} L_{\xi}^{2} L_{\tau}^{1}}\left\|g_{k}^{K^{c}}\right\|_{\langle\xi\rangle^{s} L_{\xi}^{2} L_{\tau}^{1}}\right)^{2} \\
& \leq \sum_{k} \sum_{j \leq k+5} 2^{-4(k-j)}\left\|f^{K^{c}}\right\|_{Y^{s}}^{2}\left\|g_{k}^{K^{c}}\right\|_{Y^{s}}^{2} \\
& \leq \sum_{k}\left(\sum_{p \geq-5} 2^{-4 p}\right)\left\|f^{K^{c}}\right\|_{Y^{s}}^{2}\left\|g_{k}^{K^{c}}\right\|_{Y^{s}}^{2} \lesssim\left\|f^{K^{c}}\right\|_{Y^{s}}^{2}\left\|g^{K^{c}}\right\|_{Y^{s}}^{2} .
\end{aligned}
$$

In order to control the behavior of $h_{k}^{\prime \prime}$, we perform a further decomposition; that is,

$$
\left(f_{i}^{K^{c}} * g_{j}^{K^{c}}\right)_{k, d}=\sum_{m, n}\left(\chi_{C_{m}} f_{i}^{K^{c}} * \chi_{C_{n}} g_{j}^{K^{c}}\right)_{k, d}
$$

Again, thanks to (3.16), in order for the summands to be nonzero, one of the following cases must hold:

- $m, n \leq d+5,|m-d| \leq 5$,

- $m, n \leq d+5,|n-d| \leq 5$,

- $m, n>d+5,|m-n| \leq 3$. 
By symmetry we can then assume

$$
\begin{aligned}
\left(f_{i}^{K^{c}} * g_{j}^{K^{c}}\right)_{k, d} & =\sum_{m, n \leq d+5 ;|m-d| \leq 5}\left(\chi_{C_{m}} f_{i}^{K^{c}} * \chi_{C_{n}} g_{j}^{K^{c}}\right)_{k, d} \\
& +\sum_{m, n \geq d+5 ;|m-n| \leq 3}\left(\chi_{C_{m}} f_{i}^{K^{c}} * \chi_{C_{n}} g_{j}^{K^{c}}\right)_{k, d}=I_{i, j, k, d}+I I_{i, j, k, d} .
\end{aligned}
$$

According to (3.4), we have the following estimate:

$$
\left\|\left(\chi_{C_{m}} f_{i}^{K^{c}} * \chi_{C_{n}} g_{j}^{K^{c}}\right)_{k, d}\right\|_{L_{\xi}^{2} L_{\tau}^{1}} \lesssim 2^{k+\frac{n+d}{2}}\left\|\chi_{C_{m}} f_{i}^{K^{c}}\right\| L_{L^{2}}\left\|\chi_{C_{n}} g_{j}^{K^{c}}\right\|_{L^{2}} .
$$

Hence,

$$
\begin{aligned}
& \left\|\frac{1-\chi_{K}}{\left\langle\tau-|\xi|^{2}\right\rangle}\left(\chi_{C_{m}} f_{i}^{K^{c}} * \chi_{C_{n}} g_{j}^{K^{c}}\right)_{k, d}\right\|_{\langle\xi\rangle^{s} L_{\xi}^{2} L_{\tau}^{1}} \\
& \lesssim 2^{\frac{-d+n}{2}} 2^{k(s+1)}\left\|\chi_{C_{m}} f_{i}^{K^{c}}\right\|_{L^{2}}\left\|\chi_{C_{n}} g_{j}^{K^{c}}\right\|_{L^{2}} \\
& \approx 2^{\frac{-d+n}{2}} 2^{(k-(i+j))(s+1)}\left\|\chi_{C_{m}} f_{i}^{K^{c}}\right\|_{\langle\xi\rangle^{s+1} L^{2}}\left\|\chi_{C_{n}} g_{j}^{K^{c}}\right\|_{\langle\xi\rangle^{s+1} L^{2}} \\
& \lesssim 2^{\frac{-d+n}{2}} 2^{(k-(i+j))(s+1)}\left\|\chi_{C_{m}} f_{i}^{K^{c}}\right\|_{Y^{s}}\left\|_{\chi_{C_{n}}} g_{j}^{K^{c}}\right\|_{Y^{s}} .
\end{aligned}
$$

Moreover,

$$
\sum_{d} \sum_{m=d-5}^{d+5} \sum_{n \leq d+5} 2^{\frac{-d+n}{2}}\left\|\chi_{C_{m}} f_{i}^{K^{c}}\right\|_{Y^{s}}\left\|\chi_{C_{n}} g_{j}^{K^{c}}\right\|_{Y^{s}} \lesssim\left\|f_{i}^{K^{c}}\right\|_{Y^{s}}\left\|g_{j}^{K^{c}}\right\|_{Y^{s}} .
$$

To see this, we can simplify the sum in $m$ and consider $m=d$. Then, the sum above becomes

$$
\begin{aligned}
& \sum_{d} \sum_{n \leq d+5} 2^{\frac{-d+n}{2}}\left\|\chi_{C_{d}} f_{i}^{K^{c}}\right\|_{Y^{s}}\left\|\chi_{C_{n}} g_{j}^{K^{c}}\right\|_{Y^{s}} \\
& =\sum_{p \geq-5} 2^{-\frac{p}{2}} \sum_{d}\left\|\chi_{C_{d}} f_{i}^{K^{c}}\right\|_{Y^{s}}\left\|\chi_{C_{p-d}} g_{j}^{K^{c}}\right\|_{Y^{s}}
\end{aligned}
$$

First applying Cauchy-Schwartz and then using the fact that $\sum_{p \geq-5} 2^{-\frac{p}{2}}$ is bounded gives us the claim. Therefore, we can conclude that

$$
\sum_{d}\left\|\frac{1-\chi_{K}}{\left\langle\tau-|\xi|^{2}\right\rangle} I_{i, j, k, d}\right\|_{\langle\xi\rangle^{s} L_{\xi}^{2} L_{\tau}^{1}} \lesssim 2^{(k-(i+j))(s+1)}\left\|f_{i}^{K^{c}}\right\|_{Y^{s}}\left\|g_{j}^{K^{c}}\right\|_{Y^{s}} .
$$

Hence,

$$
\begin{aligned}
& \sum_{k} \sum_{i, j \geq 5 ;|i-j| \leq 3} \sum_{d}\left\|\frac{1-\chi_{K}}{\left\langle\tau-|\xi|^{2}\right\rangle} I_{i, j, k, d}\right\|_{\langle\xi\rangle^{s} L_{\xi}^{2} L_{\tau}^{1}} \\
& \leq \sum_{k} \sum_{i, j \geq 5 ;|i-j| \leq 3} 2^{(k-(i+j))(s+1)}\left\|f_{i}^{K^{c}}\right\|_{Y^{s}}\left\|g_{j}^{K^{c}}\right\|_{Y^{s}} \lesssim\left\|f^{K^{c}}\right\| Y_{Y^{s}}\left\|g^{K^{c}}\right\|_{Y^{s}} .
\end{aligned}
$$

Now we treat the term involving $I I_{i, j, k, d}$. For this purpose we will need to use the weight $w$. We have the estimate

$$
\left\|\left(\chi_{C_{m}} f_{i}^{K^{c}} * \chi_{C_{n}} g_{j}^{K^{c}}\right)_{k, d}\right\|_{L_{\xi}^{2} L_{\tau}^{1}} \leq 2^{k+d}\left\|\chi_{C_{m}} f_{i}^{K^{c}}\right\|_{L^{2}}\left\|\chi_{C_{n}} g_{j}^{K^{c}}\right\|_{L^{2}}
$$


If $\left(\xi_{1}, \tau_{1}\right) \in A_{i} \cap C_{m},\left(\xi_{2}, \tau_{2}\right) \in A_{j} \cap C_{n}$, then $\left(\xi_{1}+\xi_{2}, \tau_{1}+\tau_{2}\right) \in A_{k} \cap B_{d}$ only if $\tau_{1}$ and $\tau_{2}$ have opposite signs. Therefore

$$
\begin{aligned}
& \left\|\frac{w}{\left\langle\tau-|\xi|^{2}\right\rangle}\left(1-\chi_{K}\right)\left(\frac{\chi_{C_{m}} f_{i}^{K^{c}}}{w} * \frac{\chi_{C_{n}} g_{j}^{K^{c}}}{w}\right)_{k, d}\right\|_{\langle\xi\rangle^{s} L_{\xi}^{2} L_{\tau}^{1}} \\
& \lesssim 2^{(k-(i+j))(s+1)} 2^{10(d-m)}\left\|\frac{\chi_{C_{m}} f_{i}^{K^{c}}}{w}\right\|\langle\xi\rangle^{s+1} L^{2}\left\|\frac{\chi_{C_{n}} g_{j}^{K^{c}}}{w}\right\|_{\langle\xi\rangle^{s+1} L^{2} .}
\end{aligned}
$$

As before, we can bound the sum:

$$
\begin{aligned}
& \sum_{d} \sum_{m, n \geq d+3 ;|m-n| \leq 3} 2^{10(d-m)}\left\|\frac{\chi_{C_{m}} f_{i}^{K^{c}}}{w}\right\|_{\langle\xi\rangle^{s+1} L^{2}}\left\|\frac{\chi_{C_{n}} g_{j}^{K^{c}}}{w}\right\|_{\langle\xi\rangle^{s+1} L^{2}} \\
& \lesssim\left\|\frac{f_{i}^{K^{c}}}{w}\right\|_{Y^{s}}\left\|\frac{g_{j}^{K^{c}}}{w}\right\|_{Y^{s}} .
\end{aligned}
$$

Hence,

$$
\begin{aligned}
& \sum_{k} \sum_{i, j \geq 5 ;|i-j| \leq 3} \sum_{d}\left\|\frac{1-\chi_{K}}{\left\langle\tau-|\xi|^{2}\right\rangle} I I_{i, j, k, d}\right\|_{\langle\xi\rangle^{s} L_{\xi}^{2} L_{\tau}^{1}} \\
& \lesssim \sum_{k} \sum_{i, j \geq 5 ;|i-j| \leq 3} 2^{(k-(i+j))(s+1)}\left\|\frac{f_{i}^{K^{c}}}{w}\right\|_{Y^{s}}\left\|\frac{g_{j}^{K^{c}}}{w}\right\|_{Y^{s}} \lesssim\left\|f^{K^{c}}\right\|_{Y^{s}}\left\|g^{K^{c}}\right\|_{Y^{s}}
\end{aligned}
$$

Combining (3.23) and (3.24), with the decompositions (3.8) and (3.22), we obtain the desired claim (3.7).

\section{The Proof of the estimate (2.16)}

This section is divided into two subsections. In the first subsection we present a preliminary result from [2, and we derive a new estimate similar to those in 2], which will be used in the next subsection. There we exhibit the proof of the bilinear estimate (2.16).

4.1. Interaction of parabolas. Here we describe how two "parabolas" interact under convolution. We will use these results in the proof of the estimate (2.16), where the two functions $f$ and $g$ are localized near the parabola $\left\{\tau \approx|\xi|^{2}\right\}$ via the indicator function $\chi_{K}$.

We remark that in the one-dimensional case, assuming that $(\xi, \tau)=\left(\xi_{1}, \tau_{1}\right)+$ $\left(\xi_{2}, \tau_{2}\right)$ one has the following resonance estimate:

$$
\max \left\{\left\langle\tau-\xi^{2}\right\rangle,\left\langle\tau_{1}-\xi_{1}^{2}\right\rangle,\left\langle\tau_{2}-\xi_{2}^{2}\right\rangle\right\} \gtrsim\left\langle\xi_{1} \xi_{2}\right\rangle .
$$

Hence it follows immediately that if both the input frequencies $\xi_{1}$ and $\xi_{2}$ are large, then it is not possible for all three $(\xi, \tau),\left(\xi_{1}, \tau_{1}\right),\left(\xi_{2}, \tau_{2}\right)$ to lie close to the parabola. This inequality is a powerful tool in the analysis of the interaction of two functions $f, g$ supported near the parabola. In higher dimensions the resonance inequality is no longer true, and we need to perform appropriate decompositions of the frequency space in order to investigate the interaction of parabolas under convolution.

The substitute for the resonance inequality will be identity,

$$
\tau-|\xi|^{2}=\tau_{1}-\left|\xi_{1}\right|^{2}+\tau_{2}-\left|\xi_{2}\right|^{2}-2 \xi_{1} \cdot \xi_{2}
$$

We introduce now a few definitions. For each $c \in \mathbb{R}$ denote by

$$
P_{c}=\left\{(\xi, \tau): \tau-|\xi|^{2}=c\right\}
$$


and by

$$
\bar{P}_{c}=\left\{(\xi, \tau): \tau+|\xi|^{2}=c\right\} .
$$

For notational simplicity let $P=P_{0}$ and $\bar{P}=\bar{P}_{0}$.

We denote by $\delta_{P_{c}}=\delta_{\tau-|\xi|^{2}=c}$ the standard surface measure associated to the parabola $P_{c}$. Thus,

$$
\delta_{P_{c}}(f)=\int f d P_{c}=\int f\left(\xi,|\xi|^{2}+c\right) \sqrt{1+4|\xi|^{2}} d \xi,
$$

for all smooth functions $f$.

Then, the $L^{2}$ norm of the restriction of a smooth function $f$ to $P_{c}$ with respect to this measure is given by

$$
\|f\|_{L^{2}\left(P_{c}\right)}=\left(\int f^{2}\left(\xi,|\xi|^{2}+c\right) \sqrt{1+4|\xi|^{2}} d \xi\right)^{\frac{1}{2}} .
$$

The following lemma can be found in 2 (see Propositions 1 and 2, section 4.1 there). Here we denote by $P^{i}$ any of the parabolas $P_{c_{i}}, \bar{P}_{c_{i}}, i=1,2$, for some constants $c_{1}, c_{2}$.

Lemma 4.1. Let $f \in L^{2}\left(P^{1}\right)$ and $g \in L^{2}\left(P^{2}\right)$ be supported on $A_{i}, A_{j}$, respectively. Then,

$$
\left\|f \delta_{P^{1}} * g \delta_{P^{2}}\right\|_{L^{2}} \leq 2^{\min (i, j)}\|f\|_{L^{2}\left(P^{1}\right)}\|g\|_{L^{2}\left(P^{2}\right)} .
$$

Moreover, if $i \leq j,\left|c_{1}\right| \leq 2^{2 i-2}$ and $\left|c_{2}\right| \leq 2^{2 j-2}$, then

$$
\left\|f \delta_{P^{1}} * g \delta_{P^{2}}\right\|_{L^{2}\left(|(\xi, \tau)| \approx 2^{j},\left.|\tau-| \xi\right|^{2} \mid \leq d\right)} \lesssim d^{\frac{1}{2}}\|f\|_{L^{2}\left(P^{1}\right)}\|g\|_{L^{2}\left(P^{2}\right)} .
$$

The next result is needed due to our particular choice of spaces.

Lemma 4.2. Let $f \in L^{2}\left(P^{1}\right)$ and $g \in L^{2}\left(P^{2}\right)$ be supported on $A_{i}, A_{j}$, respectively. Assume that $\left|c_{1}\right| \leq 2^{2 i+10}$ and $\left|c_{2}\right| \leq 2^{2 j+10}$. Then,

$$
\left\|f \delta_{P^{1}} * g \delta_{P^{2}}\right\|_{L_{\xi}^{2} L_{\tau}^{1}\left(|\xi| \approx 2^{k}\right)} \lesssim 2^{k+\frac{i+j}{2}}\|f\|_{L^{2}\left(P^{1}\right)}\|g\|_{L^{2}\left(P^{2}\right)} .
$$

Proof. Without loss of generality we can assume $c_{1}=c_{2}=0$. Also, let us consider the case when $P^{1}=P$ and $P^{2}=P$. The other cases can be treated in a similar way.

Assuming that $f \delta_{P} * g \delta_{P} \in L_{\xi}^{2} L_{\tau}^{1}\left(|\xi| \approx 2^{k}\right)$, its norm is then controlled by estimating $\left|\left(f \delta_{P} * g \delta_{P}\right) h\right|$ for any $h \in L_{\xi}^{2} L_{\tau}^{\infty}$ supported at frequency $2^{k}$. For any such $h$ we have

$$
\left(f \delta_{P} * g \delta_{P}\right) h=\int f(\xi) g(\eta) h\left(\xi+\eta,|\xi|^{2}+|\eta|^{2}\right) \sqrt{1+4|\xi|^{2}} \sqrt{1+4|\eta|^{2}} d \xi d \eta .
$$

We decompose $\mathbb{R}^{2}=\bigcup_{l \in \mathbb{Z}^{2}} Q_{l}^{k}$ in cubes $Q_{l}^{k}$ centered at $2^{k} l$ and of size $2^{k}$. Then we split

$$
f=\sum_{l \in \mathbb{Z}^{2}} f_{l} \quad \text { and } \quad g=\sum_{l \in \mathbb{Z}^{2}} g_{l}
$$

where $f_{l}$ is the part of $f$ localized in $Q_{l}^{k}$ and similarly for $g_{l}$. Since $h$ is supported at frequency $2^{k}$ we obtain

$$
\left(f \delta_{P} * g \delta_{P}\right) h=\sum_{l \in \mathbb{Z}^{2}} \int f_{l}(\xi) g_{-l}(\eta) h\left(\xi+\eta,|\xi|^{2}+|\eta|^{2}\right) \sqrt{1+4|\xi|^{2}} \sqrt{1+4|\eta|^{2}} d \xi d \eta .
$$


For fixed $l$ we evaluate

$$
\begin{aligned}
\left|\left(f_{l} \delta_{P} * g_{-l} \delta_{P}\right) h\right| & \leq\left\|f_{l}\right\|_{L^{2}(P)}|| g_{-l} \|_{L^{2}(P)} \\
& \times\left(\int h^{2}\left(\xi+\eta,|\xi|^{2}+|\eta|^{2}\right) \sqrt{1+4|\xi|^{2}} \sqrt{1+4|\eta|^{2}} d \xi d \eta\right)^{\frac{1}{2}} .
\end{aligned}
$$

Since $h \in L_{\xi}^{2} L_{\tau}^{\infty}$ we can suppose that we have $\tilde{h} \in L_{\xi}^{2}$ and estimate:

$$
\int \tilde{h}^{2}(\xi+\eta) \sqrt{1+4|\xi|^{2}} \sqrt{1+4|\eta|^{2}} d \xi d \eta \leq 2^{2 k+i+j}\|\tilde{h}\|_{L^{2}}^{2}
$$

from which we can conclude

$$
\left|\left(f_{l} \delta_{P} * g_{-l} \delta_{P}\right) h\right| \leq 2^{k+\frac{i+j}{2}}|| f_{l}\left\|_{L^{2}(P)}|| g_{-l}\right\|_{L^{2}(P)}|| h \|_{L_{\xi}^{2} L_{\tau}^{\infty}} .
$$

Summing up with respect to $l$ gives us the claim in (4.5), as long as $f \delta_{P} * g \delta_{P} \in$ $L_{\xi}^{2} L_{\tau}^{1}\left(|\xi| \approx 2^{k}\right)$. In order to guarantee this last fact, we can pick $h \in L_{\xi, \tau}^{2}$ and perform a similar computation to the one above. In this way we obtain an $L^{2}$ estimate for $f \delta_{P} * g \delta_{P}$. However, $L_{\xi, \tau}^{2}\left(|\xi| \approx 2^{k}\right) \subset L_{\xi}^{2} L_{\tau}^{1}\left(|\xi| \approx 2^{k}\right)$, and this concludes the proof.

4.2. Proof of the bilinear estimate (2.16). We start with an auxiliary lemma.

Lemma 4.3. Let $f, g$ be nonnegative smooth functions. Assume that $0 \leq i \leq$ $j, d_{1} \leq 2 i-4, d_{2} \leq 2 j-4$. Then, for $|k-j| \leq 5$ and $d_{3} \leq 2 k-4$, the following estimate holds:

$$
\|\left.\left(f_{i, d_{1}} * g_{j, d_{2}}\right)_{k, d_{3}}\right|_{\hat{X}^{0,-\frac{1}{2}}} \lesssim 2^{-\frac{i+j}{2}}|| f_{i, d_{1}}||_{\hat{X}^{0, \frac{1}{2}}}|| g_{j, d_{2}}||_{\hat{X}^{0, \frac{1}{2}}} .
$$

Moreover, if $|i-j| \leq 2, k \leq j+2, d_{1} \leq 2 i-4$ and $d_{2} \leq 2 j-4$, then

$$
\begin{gathered}
\left\|f_{i, d_{1}} * g_{j, d_{2}}\right\|_{L^{2}} \leq\left\|f_{i, d_{1}}\right\|_{\hat{X}^{0, \frac{1}{2}}}\left\|g_{j, d_{2}}\right\|_{\hat{X}^{0, \frac{1}{2}}}, \\
\left\|\left(f_{i, d_{1}} * g_{j, d_{2}}\right)_{k}\right\|_{L_{\xi}^{2} L_{\tau}^{1}} \lesssim 2^{k}\left\|f_{i, d_{1}}\right\|_{\hat{X}^{0, \frac{1}{2}}}\left\|g_{j, d_{2}}\right\|_{\hat{X}^{0, \frac{1}{2}}} .
\end{gathered}
$$

Proof. The proof of (4.6) and (4.7) can be found in [2]; see the proof of Proposition 3 , section 4.2. These estimates can also be derived directly from the statements of Proposition 3, section 4.2 in [2].

Thus, we are left with the proof of (4.8), which is specific to our problem. We aim to apply the results in Subsection 4.1. Notice that on each $A_{i}$ the parabolas $P_{c}$ make an angle of approximately $2^{-i}$ with the $\tau$ axis. Thus, recalling (4.2), we have the following relation between measures:

$$
d \xi d \tau \approx 2^{-i} d P_{c} d c .
$$

Therefore for each $h$ supported in $A_{k} \cap B_{d}$ we have

$$
\|h\|_{\hat{X}^{0, \pm \frac{1}{2}}}^{2} \approx 2^{ \pm d} \int_{2^{d-1}}^{2^{d+1}}\|h\|_{L^{2}\left(P_{b}\right)}^{2} 2^{-k} d b .
$$


For notational simplicity, we denote $f=f_{i, d_{1}}, g=g_{j, d_{2}}$. We apply (4.5) and (4.9) to evaluate the following norm:

$$
\begin{aligned}
& \|f * g\|_{L_{\xi}^{2} L_{\tau}^{1}} \leq \int_{I_{1}} \int_{I_{2}}\left\|f \delta_{P_{b_{1}}} * g \delta_{P_{b_{2}}}\right\|_{L_{\xi}^{2} L_{\tau}^{1}} 2^{-i-j} d b_{1} d b_{2} \\
& \leq \int_{I_{1}} \int_{I_{2}} 2^{k-\frac{i+j}{2}}\|f\|_{L^{2}\left(P_{b_{1}}\right)}\|g\|_{L^{2}\left(P_{b_{2}}\right)} d b_{1} d b_{2} \\
& \leq 2^{k}\left(\int_{I_{1}}\left(1+b_{1}\right)^{-1} d b_{1}\right)^{\frac{1}{2}}\|f\|_{\hat{X}^{0, \frac{1}{2}}}\left(\int_{I_{2}}\left(1+b_{2}\right)^{-1} d b_{2}\right)^{\frac{1}{2}}\|g\|_{\hat{X}^{0, \frac{1}{2}}} \\
& \approx 2^{k}\|f\|_{\hat{X}^{0, \frac{1}{2}}}\|g\|_{\hat{X}^{0, \frac{1}{2}}} .
\end{aligned}
$$

Here we used the fact that $I_{1} \approx\left[2^{d_{1}-1}, 2^{d_{1}+1}\right]$, which gives us $\int\left(1+b_{1}\right)^{-1} d b_{1} \approx 1$. The same thing applies for the integral with respect to $b_{2}$.

We remark that the proof of (4.6)-(4.7) follows the same lines as the proof above, and uses Lemma 4.1, which we have thus stated for the convenience of the reader. However, the proof is slightly more involved, hence we prefer to refer the reader to [2] as well.

Proof of (2.16). We wish to prove that

$$
\left\|\frac{w}{\left\langle\tau-|\xi|^{2}\right\rangle}\left(\frac{f^{K}}{w} * \frac{g^{K}}{w}\right)\right\|_{Z^{s}} \lesssim\left\|f^{K}\right\|_{\hat{X}^{s, \frac{1}{2}, 1}}\left\|g^{K}\right\|_{\hat{X}^{s, \frac{1}{2}, 1}} .
$$

On behalf of (2.19) we reduce to showing the following bound:

$$
\left\|\frac{1}{\left\langle\tau-|\xi|^{2}\right\rangle}\left(f^{K} * g^{K}\right)\right\|_{Z^{s}} \lesssim\left\|f^{K}\right\|_{\hat{X}^{s, \frac{1}{2}, 1}}\left\|g^{K}\right\|_{\hat{X}^{s, \frac{1}{2}, 1}} .
$$

Now, we assume by symmetry that $i \leq j$; we perform the following decomposition:

$$
\left(f_{i}^{K} * g_{j}^{K}\right)=\chi_{K}\left(f_{i}^{K} * g_{j}^{K}\right)+\left(1-\chi_{K}\right)\left(f_{i}^{K} * g_{j}^{K}\right) .
$$

We continue with decomposing even further the first term, that is,

$$
\chi_{K}\left(f_{i}^{K} * g_{j}^{K}\right)=\sum_{k} \sum_{d_{3} \leq 2 k-4} \sum_{d_{1} \leq 2 i-4} \sum_{d_{2} \leq 2 j-4}\left(f_{i, d_{1}} * g_{j, d_{2}}\right)_{k, d_{3}} .
$$

By the definition of $K$, in support of $f_{i}^{K}$ we have $\tau \geq 2^{2 i-4}$ while in the support of $g_{j}^{K}$ we have $\tau \geq 2^{2 j-4}$. Hence, in the support of $f_{i}^{K} * g_{j}^{K}$ we have $\tau \geq 2^{2 j-4}$ and in the support of $\chi_{K}\left(f_{i}^{K} * g_{j}^{K}\right)$ we have $|\xi| \geq 2^{j-5}$. This means we have nontrivial interactions only in the case $|k-j| \leq 5$. 
Hence, using (4.6) together with (4.12), we get the following estimate:

$$
\begin{aligned}
& \left\|\frac{\chi_{K}}{\left\langle\tau-|\xi|^{2}\right\rangle}\left(f_{i}^{K} * g_{j}^{K}\right)\right\|_{\hat{X}^{0, \frac{1}{2}, 1}} \approx\left\|\chi_{K}\left(f_{i}^{K} * g_{j}^{K}\right)\right\|_{\hat{X}^{0,-\frac{1}{2}, 1}} \\
& \lesssim \sum_{|k-j| \leq 5} \sum_{d_{3} \leq 2 k-4} \sum_{d_{1} \leq 2 i-4} \sum_{d_{2} \leq 2 j-4}\left\|\left(f_{i, d_{1}} * g_{j, d_{2}}\right)_{k, d_{3}}\right\|_{\hat{X}^{0,-\frac{1}{2}, 1}} \\
& \lesssim \sum_{|k-j| \leq 5} \sum_{d_{3} \leq 2 k-4} \sum_{d_{1} \leq 2 i-4} \sum_{d_{2} \leq 2 j-4} 2^{-\frac{i+j}{2}}\left\|f_{i, d_{1}}\right\|_{\hat{X}^{0, \frac{1}{2}}}\left\|g_{j, d_{2}}\right\|_{\hat{X}^{0, \frac{1}{2}}} \\
& \lesssim 2^{-\frac{i+j}{2}} \sum_{|k-j| \leq 5} \sum_{d_{3} \leq 2 k-4}\left\|f_{i}^{K}\right\|_{\hat{X}^{0, \frac{1}{2}, 1}}\left\|g_{j}^{K}\right\|_{\hat{X}^{0, \frac{1}{2}, 1}} \\
& \lesssim j 2^{-\frac{i+j}{2}}\left\|f_{i}^{K}\right\|_{\hat{X}^{0, \frac{1}{2}, 1}}\left\|g_{j}^{K}\right\|_{\hat{X}^{0, \frac{1}{2}, 1}} .
\end{aligned}
$$

For general $s$ this becomes

$$
\left\|\frac{\chi_{K}}{\left\langle\tau-|\xi|^{2}\right\rangle}\left(f_{i}^{K} * g_{j}^{K}\right)\right\|_{\hat{X}^{s, \frac{1}{2}, 1}} \leq j 2^{-(1+s) i} 2^{\frac{i-j}{2}}\left\|f_{i}^{K}\right\|_{\hat{X}^{s, \frac{1}{2}, 1}}\left\|g_{j}^{K}\right\|_{\hat{X}^{s, \frac{1}{2}, 1}} .
$$

Summing up with respect to $i, j$ gives us

$$
\begin{aligned}
& \left\|\frac{\chi_{K}}{\left\langle\tau-|\xi|^{2}\right\rangle}\left(f^{K} * g^{K}\right)\right\|_{X^{s, \frac{1}{2}, 1}} \\
& \lesssim \sum_{i \leq j} j 2^{-(1+s) i} 2^{\frac{i-j}{2}}\left\|f_{i}^{K}\right\|_{\hat{X}^{s, \frac{1}{2}, 1}}\left\|g_{j}^{K}\right\|_{\hat{X}^{s, \frac{1}{2}, 1}}+\text { symmetric term } \\
& \lesssim\left\|f^{K}\right\|_{\hat{X}^{s, \frac{1}{2}, 1}}\left\|g^{K}\right\|_{\hat{X}^{s, \frac{1}{2}, 1}} .
\end{aligned}
$$

Finally, since $\hat{X}^{s, \frac{1}{2}, 1}$ and $Z^{s}$ paste nicely in the set $K$, we have obtained that

$$
\left\|\frac{\chi_{K}}{\left\langle\tau-|\xi|^{2}\right\rangle}\left(f^{K} * g^{K}\right)\right\|_{Z^{s}} \lesssim\left\|f^{K}\right\|_{\hat{X}^{s, \frac{1}{2}, 1}}\left\|g^{K}\right\|_{\hat{X}^{s, \frac{1}{2}, 1}} \text {. }
$$

For the second term in (4.11) we make the following observation. If $i \leq j-5$, then $f_{i}^{K} * g_{j}^{K}$ is supported in $\bigcup_{k=j-1}^{j+1} A_{k} \cap B_{\leq 2 j-3}$. Hence, by the pasting lemma, in this case the $Z^{s}$ norm of $\left(1-\chi_{K}\right)\left(f_{i}^{K} * g_{j}^{K}\right)$ is controlled by the $\hat{X}^{s, \frac{1}{2}, 1}$ norm. Morally, in this case we keep the interaction close enough to $P$ so that we can treat the estimate in the same way as the previous one. Indeed, using the same computations as above we obtain

$$
\sum_{|i-j| \geq 5}\left\|\frac{1-\chi_{K}}{\left\langle\tau-|\xi|^{2}\right\rangle}\left(f_{i}^{K} * g_{j}^{K}\right)\right\|_{\hat{X}^{s, \frac{1}{2}, 1}} \lesssim\left\|f^{K}\right\|_{\hat{X}^{s, \frac{1}{2}, 1}}\left\|g^{K}\right\|_{\hat{X}^{s, \frac{1}{2}, 1}}
$$

In the case when $|i-j| \leq 4$ we perform the following decomposition:

$$
\left(1-\chi_{K}\right)\left(f_{i}^{K} * g_{j}^{K}\right)=\sum_{k} \sum_{d_{1} \leq 2 i-4} \sum_{d_{2} \leq 2 j-4}\left(1-\chi_{K}\right)\left(f_{i, d_{1}} * g_{j, d_{2}}\right)_{k} .
$$

We notice that $f_{i}^{K} * g_{j}^{K}$ is supported in a region where $|\xi| \leq 2^{j+10}, \tau \geq 2^{2 j-4}$ and $\left.|\tau-| \xi\right|^{2} \mid \geq 2^{2 j-10}$. Using the decomposition (4.16) and the estimate (4.7) we obtain

$$
\begin{aligned}
& \sum_{|i-j| \leq 4}\left\|\frac{1-\chi_{K}}{\left\langle\tau-|\xi|^{2}\right\rangle}\left(f_{i}^{K} * g_{j}^{K}\right)\right\|_{\langle(\xi, \tau)\rangle^{s+1} L^{2}} \\
& \lesssim \sum_{|i-j| \leq 4} \sum_{k \leq j+10} 2^{(s+1)(k-2 j)}\left\|f_{i}^{K}\right\|_{\hat{X}^{s, \frac{1}{2}, 1}}\left\|g_{j}^{K}\right\|_{\hat{X}^{s, \frac{1}{2}, 1}} \\
& \lesssim\left\|f^{K}\right\|_{\hat{X}^{s, \frac{1}{2}, 1}}\left\|g^{K}\right\|_{\hat{X}^{s, \frac{1}{2}, 1}},
\end{aligned}
$$


while using the estimate (4.8) we get

$$
\begin{aligned}
& \sum_{|i-j| \leq 4}\left\|\frac{1-\chi_{K}}{\left\langle\tau-|\xi|^{2}\right\rangle}\left(f_{i}^{K} * g_{j}^{K}\right)\right\|_{\langle\xi\rangle^{s} L_{\xi}^{2} L_{\tau}^{1}} \\
& \lesssim \sum_{|i-j| \leq 4} \sum_{k \leq j+10} 2^{(s+1)(k-2 j)}\left\|f_{i}^{K}\right\|_{\hat{X}^{s, \frac{1}{2}, 1}}\left\|g_{j}^{K}\right\|_{\hat{X}^{s, \frac{1}{2}, 1}} \\
& \lesssim\left\|f^{K}\right\|_{\hat{X}^{s, \frac{1}{2}, 1}}\left\|g^{K}\right\|_{\hat{X}^{s, \frac{1}{2}, 1}} .
\end{aligned}
$$

The estimates (4.15), (4.17) and (4.18), the definition of the space $Y^{s}$, and the fact that $Y^{s}$ and $Z^{s}$ paste nicely outside of $K$ then imply

$$
\left\|\frac{1-\chi_{K}}{\left\langle\tau-|\xi|^{2}\right\rangle}\left(f^{K} * g^{K}\right)\right\|_{Z^{s}} \lesssim\left\|f^{K}\right\|_{\hat{X}^{s, \frac{1}{2}, 1}}\left\|g^{K}\right\|_{\hat{X}^{s, \frac{1}{2}, 1}} .
$$

Combining (4.14) and (4.19) we get the claim in (4.10).

\section{The PRoof of The EStimate (2.17)}

In this section we present the proof of the bilinear estimate (2.17). We start by proving an auxiliary lemma.

Lemma 5.1. Let $f, g$ be nonnegative smooth functions. If $k \geq i-10, d_{3} \leq 2 k-4$ and $d_{1} \leq 2 i-4$, then

$$
\left\|\left(f_{i, d_{1}} * g\right)_{k, d_{3}}\right\|_{\hat{X}^{0,-\frac{1}{2}}} \lesssim 2^{\frac{i-k}{2}}\left\|f_{i, d_{1}}\right\|_{\hat{X}^{0, \frac{1}{2}}}\|g\|_{L^{2}} .
$$

If $d_{2} \leq 2 i-10, d_{3} \leq 2 k-4$ and $d_{1} \leq 2 i-4$, then

$$
\left\|\left(f_{i, d_{1}} * g_{d_{2}}^{K^{c}}\right)_{k, d_{3}}\right\|_{\hat{X}^{0,-\frac{1}{2}}} \lesssim 2^{\frac{3 d_{2}-2 i-2 d_{3}}{4}}\left\|f_{i, d_{1}}\right\|_{\hat{X}^{0, \frac{1}{2}}}\left\|g_{d_{2}}^{K^{c}}\right\|_{L^{2}}
$$

If $k \leq i-10$ and $d_{3} \leq 2 k-4$ and $d_{1} \leq 2 i-4$, then

$$
\left\|\left(f_{i, d_{1}} * \frac{g}{w}\right)_{k, d_{3}}\right\|_{\hat{X}^{0,-\frac{1}{2}}} \lesssim 2^{-4 i}\left\|f_{i, d_{1}}\right\|_{\hat{X}^{0, \frac{1}{2}}}\|g\|_{L^{2}}
$$

Proof. By duality, (5.1) is equivalent to

$$
\left\|f_{i, d_{1}} * g_{k, d_{3}}\right\|_{L^{2}} \lesssim 2^{\frac{i-k}{2}}\left\|f_{i, d_{1}}\right\|_{\hat{X}^{0, \frac{1}{2}}}\left\|g_{k, d_{3}}\right\|_{\hat{\bar{X}}^{0, \frac{1}{2}}}
$$

which can be obtained by a similar argument to the one for (4.6), in light of the fact that (4.3) allows us to work with either $\delta_{P_{c}}$ or $\delta_{\bar{P}_{c}}$. For a detailed argument we refer to [2].

For the proof of (5.2) we split $A_{i} \cap B_{d_{1}}=\bigcup_{\alpha} D_{\alpha}$, where $D_{\alpha}$ are disjoint sets of sizes $2^{-i} 2^{d_{1}} \times 2^{\frac{d_{2}}{2}} \times 2^{d_{2}}$, the first size being in the direction of $n_{\alpha}$ and the last one in the direction of $\tau$. Here by $n_{\alpha}$ we mean one of the normal directions to $P_{d_{2}} \cap D_{\alpha}$.

We replace $f_{i, d_{1}}$ by $f$, so that we do not carry all the indexes.

Then, if $f_{\alpha}$ is the part of $f$ localized in $D_{\alpha}$, the $f_{\alpha} * g$ 's have, essentially, disjoint support with respect to $\alpha$. Hence,

$$
\begin{gathered}
\|f * g\|_{L^{2}}^{2} \approx \sum_{\alpha}\left\|f_{\alpha} * g\right\|_{L^{2}}^{2} \leq \sum_{\alpha}\left\|f_{\alpha}\right\|_{L^{1}}^{2}\|g\|_{L^{2}}^{2} \\
\lesssim 2^{-i+d_{1}} 2^{\frac{3}{2} d_{2}} \sum_{\alpha}\left\|f_{\alpha}\right\|_{L^{2}}^{2}\|g\|_{L^{2}}^{2} \lesssim 2^{-i+d_{1}} 2^{\frac{3}{2} d_{2}}\|f\|_{L^{2}}^{2}\|g\|_{L^{2}}^{2} .
\end{gathered}
$$


Thus,

$$
\begin{aligned}
\left\|(f * g)_{j, d_{3}}\right\|_{\hat{X}^{0,-\frac{1}{2}}} & \lesssim 2^{-\frac{d_{3}}{2}}\|f * g\|_{L^{2}} \lesssim 2^{\frac{3 d_{2}-2 i-2 d_{3}}{4}} 2^{\frac{d_{1}}{2}}\|f\|_{L^{2}}\|g\|_{L^{2}} \\
& \approx 2^{\frac{3 d_{2}-2 i-2 d_{3}}{4}}\left\|f_{i, d_{1}}\right\|_{\hat{X}^{0, \frac{1}{2}}}\|g\|_{L^{2}}
\end{aligned}
$$

that is the desired estimate.

We start the proof of (5.3) with a geometrical observation. The function $f$ is supported in a region where $\tau \geq 2^{2 i-4}$ and we want to restrict $f * g$ in a region where $\tau \leq 2^{2 k+4}$. This can be achieved only by restricting the support of $g$ in a region where $\tau \leq-2^{2 i-5}$. This allows us to obtain pretty loose estimates, thanks to the weight $w$.

Indeed, we can run the $L^{1} * L^{2} \rightarrow L^{2}$ argument to get

$$
\left\|\left(f * \frac{g}{w}\right)_{k, d_{3}}\right\|_{\hat{X}^{0,-\frac{1}{2}}} \lesssim\left\|f * \frac{g}{w}\right\|_{L^{2}} \lesssim\|f\|_{L^{1}}\left\|\frac{g}{w}\right\|_{L^{2}} \lesssim 2^{i}\left\|f_{i, d_{1}}\right\|_{\hat{X}^{0, \frac{1}{2}}} 2^{-20 i}\|g\|_{L^{2}} .
$$

This concludes our proof.

Proof of (2.17). We wish to prove that

$$
\left\|\frac{\chi_{K} w}{\left\langle\tau-|\xi|^{2}\right\rangle}\left(\frac{f^{K}}{w} * \frac{g^{K^{c}}}{w}\right)\right\|_{\hat{X}^{s, \frac{1}{2}, 1}} \lesssim\left\|f^{K}\right\|_{\hat{X}^{s, \frac{1}{2}, 1}}\left\|g^{K^{c}}\right\|_{Y^{s}}
$$

Since $w=1$ in $K$, we can drop $w$ from $f^{K} / w$. Then we decompose

$$
\begin{aligned}
& \chi_{K}\left(f^{K} * \frac{g^{K^{c}}}{w}\right)=\sum_{k} \sum_{d_{3} \leq 2 k-4} \sum_{i} \sum_{d_{1} \leq 2 i-4} \sum_{d_{2}}\left(f_{i, d_{1}} * \frac{g_{d_{2}}^{K^{c}}}{w}\right)_{k, d_{3}} \\
& =\sum_{k} \sum_{d_{3} \leq 2 k-4} \sum_{i \leq k+10} \sum_{d_{1} \leq 2 i-4} \sum_{d_{2}}\left(f_{i, d_{1}} * \frac{g_{d_{2}}^{K^{c}}}{w}\right)_{k, d_{3}} \\
& +\sum_{k} \sum_{d_{3} \leq 2 k-4} \sum_{i \geq k+11} \sum_{d_{1} \leq 2 i-4} \sum_{d_{2}}\left(f_{i, d_{1}} * \frac{g_{d_{2}}^{K^{c}}}{w}\right)_{k, d_{3}} \\
& =I_{1}+I_{2} .
\end{aligned}
$$

We decompose $I_{1}$ even further

$$
\begin{aligned}
I_{1} & =\sum_{k} \sum_{d_{3} \leq 2 k-4} \sum_{i \leq k+10} \sum_{d_{1} \leq 2 i-4} \sum_{d_{2} \geq \frac{i}{10}}\left(f_{i, d_{1}} * \frac{g_{d_{2}}^{K^{c}}}{w}\right)_{k, d_{3}} \\
& +\sum_{k} \sum_{d_{3} \leq 2 k-4} \sum_{i \leq k+10} \sum_{d_{1} \leq 2 i-4} \sum_{d_{2}<\frac{i}{10}}\left(f_{i, d_{1}} * \frac{g_{d_{2}}^{K^{c}}}{w}\right)_{k, d_{3}} \\
& =I_{11}+I_{12} .
\end{aligned}
$$

We use (5.1) to estimate $I_{11}$ :

$$
\begin{aligned}
& \left\|\frac{1}{\left\langle\tau-|\xi|^{2}\right\rangle} I_{11}\right\|_{X^{s, \frac{1}{2}, 1}} \lesssim\left\|I_{11}\right\|_{X^{s,-\frac{1}{2}, 1}} \\
& \lesssim \sum_{k} \sum_{d_{3} \leq 2 k-4} \sum_{i \leq k+10} \sum_{d_{1} \leq 2 i-4} \sum_{d_{2} \geq \frac{i}{10}} 2^{k s}\left\|\left(f_{i, d_{1}} * g_{d_{2}}^{K^{c}}\right)_{k, d_{3}}\right\|_{\hat{X}^{0,-\frac{1}{2}}} \\
& \lesssim \sum_{k} \sum_{d_{3} \leq 2 k-4} \sum_{i \leq k+10} \sum_{d_{1} \leq 2 i-4} \sum_{d_{2} \geq \frac{i}{10}} 2^{k s} 2^{\frac{i-k}{2}}\left\|f_{i, d_{1}}\right\|_{\hat{X}^{0, \frac{1}{2}}}\left\|g_{d_{2}}^{K^{c}}\right\|_{L^{2}}
\end{aligned}
$$




$$
\begin{aligned}
& \lesssim \sum_{k} \sum_{d_{3} \leq 2 k-4} \sum_{i \leq k+10} \sum_{d_{2} \geq \frac{i}{10}} 2^{k s} 2^{\frac{i-k}{2}} 2^{-\frac{(s+1) d_{2}}{2}}\left\|f_{i}^{K}\right\|_{\hat{X}^{0, \frac{1}{2}, 1}}\left\|g_{d_{2}}^{K^{c}}\right\|_{Y^{s}} \\
& \lesssim \sum_{k} \sum_{d_{3} \leq 2 k-4} \sum_{i \leq k+10} 2^{(k-i) s} 2^{\frac{i-k}{2}} 2^{-(s+1) \frac{i}{20}}\left\|f_{i}^{K}\right\|_{\hat{X}^{s, \frac{1}{2}, 1}}\left\|\chi_{B_{\geq \frac{i}{10}}} g^{K^{c}}\right\|_{Y^{s}} \\
& \lesssim \sum_{k} \sum_{i \leq k+10} k 2^{(k-i)\left(s-\frac{1}{2}\right)} 2^{-(s+1) \frac{i}{20}}\left\|f_{i}^{K}\right\|_{\hat{X}^{s, \frac{1}{2}, 1}}\left\|g^{K^{c}}\right\|_{Y^{s}} \lesssim\left\|f^{K}\right\|_{\hat{X}^{s, \frac{1}{2}, 1}}\left\|g^{K^{c}}\right\|_{Y^{s}}
\end{aligned}
$$

When estimating $I_{12}$ we notice that unless $|k-i| \leq 2$ we have trivial estimates. Indeed the support of $\chi_{B_{\leq \frac{i}{10}}} g^{K^{c}}$ cannot move, via convolution, the support of $f_{i}$ too much. We use (5.2) and compute

$$
\begin{aligned}
& \left\|\frac{1}{\left\langle\tau-|\xi|^{2}\right\rangle} I_{12}\right\|_{X^{s, \frac{1}{2}, 1}} \lesssim\left\|I_{12}\right\|_{X^{s,-\frac{1}{2}, 1}} \\
& \lesssim \sum_{k} \sum_{d_{3} \leq 2 k-4} \sum_{i=k-2}^{k+2} \sum_{d_{1} \leq 2 i-4} \sum_{d_{2} \leq \frac{i}{10}} 2^{k s}\left\|\left(f_{i, d_{1}} * g_{d_{2}}^{K^{c}}\right)_{k, d_{3}}\right\|_{\hat{X}^{0,-\frac{1}{2}}} \\
& \lesssim \sum_{k} \sum_{d_{3} \leq 2 k-4} \sum_{i=k-2}^{k+2} \sum_{d_{1} \leq 2 i-4} \sum_{d_{2} \leq \frac{i}{10}} 2^{k s} 2^{\frac{3 d_{2}-2 i-2 d_{3}}{4}}\left\|f_{i, d_{1}}\right\|_{\hat{X}^{0, \frac{1}{2}}}\left\|g_{d_{2}}^{K^{c}}\right\|_{L^{2}} \\
& \lesssim \sum_{k} \sum_{d_{3} \leq 2 k-4} \sum_{i=k-2}^{k+2} \sum_{d_{2} \leq \frac{i}{10}} 2^{\frac{3 d_{2}-2 i-2 d_{3}}{4}} 2^{-\frac{(s+1) d_{2}}{2}}\left\|f_{i}^{K}\right\|_{\hat{X}^{s, \frac{1}{2}, 1}}\left\|g_{d_{2}}^{K^{c}}\right\|_{Y^{s}} \\
& \lesssim \sum_{k} \sum_{d_{3} \leq 2 k-4} \sum_{i=k-2}^{k+2} 2^{\frac{-i-2 d_{3}}{4}}\left\|f_{i}^{K}\right\|_{\hat{X}^{s, \frac{1}{2}, 1}}\left\|\chi_{X_{\leq}} g^{K^{c}}\right\|_{Y^{s}} \\
& \lesssim \sum_{k} \sum_{i=k-2}^{k+2} 2^{-\frac{i}{4}}\left\|f_{i}^{K}\right\|_{\hat{X}^{s, \frac{1}{2}, 1}}\|g\|_{Y^{s}} \lesssim\left\|f^{K}\right\|_{\hat{X}^{s, \frac{1}{2}, 1}}\left\|g^{K^{c}}\right\|_{Y^{s}} .
\end{aligned}
$$

Finally, we use (5.3) to estimate $I_{2}$ :

$$
\begin{aligned}
& \left\|\frac{1}{\left\langle\tau-|\xi|^{2}\right\rangle} I_{2}\right\|_{X^{s, \frac{1}{2}, 1}} \lesssim\left\|I_{2}\right\|_{X^{s,-\frac{1}{2}, 1}} \\
& \lesssim \sum_{k} \sum_{d_{3} \leq 2 k-4} \sum_{i \geq k+11} \sum_{d_{1} \leq 2 i-4} 2^{k s}\left\|\left(f_{i, d_{1}} * \frac{g^{K^{c}}}{w}\right)_{k, d_{3}}\right\|_{\hat{X}^{0,-\frac{1}{2}}} \\
& \lesssim \sum_{k} \sum_{d_{3} \leq 2 k-4} \sum_{i \geq k+11} \sum_{d_{1} \leq 2 i-4} 2^{k s} 2^{-4 i}\left\|f_{i, d_{1}}\right\|_{\hat{X}^{0, \frac{1}{2}}}\left\|g^{K^{c}}\right\|_{L^{2}} \\
& \lesssim \sum_{k} \sum_{d_{3} \leq 2 k-4} \sum_{i \geq k+11} 2^{k s} 2^{-4 i}\left\|f_{i}\right\|_{\hat{X}^{0, \frac{1}{2}, 1}}\left\|g^{K^{c}}\right\|_{Y^{s}} \\
& \lesssim \sum_{k} k 2^{k s} \sum_{i \geq k+11} 2^{-3 i}\left\|f_{i}\right\|_{\hat{X}^{s, \frac{1}{2}, 1}}\left\|g^{K^{c}}\right\|_{Y^{s}} \lesssim\|f\|_{\hat{X}^{s, \frac{1}{2}, 1}}\left\|g^{K^{c}}\right\|_{Y^{s}} .
\end{aligned}
$$

Adding up the estimates that we obtained for $I_{11}, I_{12}$ and $I_{2}$ gives us the estimate (2.17). 


\section{The PRoof of the Estimate 2.18)}

We start with the following two elementary auxiliary lemmas.

Lemma 6.1. Let $f, g$ be nonnegative smooth functions. Then,

$$
\begin{gathered}
\left\|f_{i} * g_{j}\right\|_{L_{\xi}^{2} L_{\tau}^{1}} \lesssim 2^{\min (i, j)}\left\|f_{i}\right\|_{L_{\xi}^{2} L_{\tau}^{1}}\left\|g_{j}\right\|_{L_{\xi}^{2} L_{\tau}^{1}}, \\
\left\|\left(f_{i} * g_{j}\right)_{k}\right\|_{L_{\xi}^{2} L_{\tau}^{1}} \lesssim 2^{k}\left\|f_{i}\right\|_{L_{\xi}^{2} L_{\tau}^{1}}\left\|g_{j}\right\|_{L_{\xi}^{2} L_{\tau}^{1}} .
\end{gathered}
$$

Moreover if $|i-j| \leq 3, k \leq \max (i, j)-10$, and $d \leq 2 \max (i, j)-10$, then

$$
\left\|\left(f_{i}^{K} * \frac{g_{j}}{w}\right)_{k, d}\right\|_{L_{\xi}^{2} L_{\tau}^{1}} \lesssim 2^{k} 2^{-20 j}\left\|f_{i}\right\|_{L_{\xi}^{2} L_{\tau}^{1}}\left\|g_{j}\right\|_{L_{\xi}^{2} L_{\tau}^{1}} .
$$

Proof. The first estimate is trivial:

$$
\left\|f_{i} * g_{j}\right\|_{L_{\xi}^{2} L_{\tau}^{1}} \lesssim\left\|f_{i}\right\|_{L_{\xi}^{1} L_{\tau}^{1}}\left\|g_{j}\right\|_{L_{\xi}^{2} L_{\tau}^{1}} \lesssim 2^{i}\left\|f_{i}\right\|_{L_{\xi}^{2} L_{\tau}^{1}}\left\|g_{j}\right\|_{L_{\xi}^{2} L_{\tau}^{1}} \cdot
$$

Analogously,

$$
\left\|f_{i} * g_{j}\right\|_{L_{\xi}^{2} L_{\tau}^{1}} \lesssim 2^{j}\left\|f_{i}\right\|_{L_{\xi}^{2} L_{\tau}^{1}}\left\|g_{j}\right\|_{L_{\xi}^{2} L_{\tau}^{1}}
$$

and (6.1) immediately follows.

For (6.2) we estimate as follows:

$$
\left\|\left(f_{i} * g_{j}\right)_{k}\right\|_{L_{\xi}^{2} L_{\tau}^{1}} \lesssim 2^{k}\left\|\left(f_{i} * g_{j}\right)_{k}\right\|_{L_{\xi}^{\infty} L_{\tau}^{1}} \lesssim 2^{k}\left\|f_{i}\right\|_{L_{\xi}^{2} L_{\tau}^{1}}\left\|g_{j}\right\|_{L_{\xi}^{2} L_{\tau}^{1}} .
$$

In order to derive (6.3) the key observation is that the part of $g_{j}$ which interacts nontrivially is localized in a region where $\tau \leq-2^{2 j-10}$. Roughly speaking, we have a high-high to low type of interaction, and $f_{i}^{K}$ is localized at $\tau \geq 2^{2 i-5}$. Using this observation, the argument to prove (6.3) is similar to the one for (6.2).

Lemma 6.2. Let $f, g$ be nonnegative smooth functions. Then

$$
\left\|f_{i} * g_{j}\right\|_{L^{2}} \lesssim 2^{\min (i, j)}\left\|f_{i}\right\|_{L_{\xi}^{2} L_{\tau}^{1}}\left\|g_{j}\right\|_{L^{2}}
$$

Moreover if $d \leq 2 i-20$, then

$$
\left\|\left(1-\chi_{K}\right)\left(f_{i}^{K} * \frac{g}{w}\right)_{d}\right\|_{L^{2}} \leq 2^{\frac{d}{2}} 2^{-20 i}\left\|f_{i}\right\|_{L_{\xi}^{2} L_{\tau}^{1}}\|g\|_{L^{2}} .
$$

Proof. The first estimate is trivial. Indeed,

$$
\left\|f_{i} * g_{j}\right\|_{L^{2}} \lesssim\left\|f_{i}\right\|_{L_{\xi}^{1} L_{\tau}^{1}}\left\|g_{j}\right\|_{L^{2}} \lesssim 2^{i}\left\|f_{i}\right\|_{L_{\xi}^{2} L_{\tau}^{1}}\left\|g_{j}\right\|_{L^{2}}
$$

and similarly we get the one with $2^{j}$ replacing $2^{i}$.

In order to derive (6.5), it is enough to observe that the part of $g$ which gives nontrivial interactions is localized in a region where $\tau \lesssim-2^{2 i-5}$. Moreover, the outcome of the interaction is localized at $|\xi| \lesssim 2^{\frac{d}{2}}$. These two facts together with a similar argument to the one in the previous lemma give us the desired estimate.

To justify our first observation, we notice that $f_{i}^{K}$ is localized at $\tau \geq 2^{2 i-5}$, while $\chi_{B_{d}}\left(1-\chi_{K}\right)$ is supported in a region where $|\tau| \leq\left. 2^{6}|\tau-| \xi\right|^{2} \mid \leq 2^{d+7} \leq 2^{2 i-10}$. As a consequence the interacting part of $g$ is supported in a region with $\tau \lesssim-2^{2 i-5}$.

To justify our second observation, we notice that the size of the support of $\chi_{B_{d}}\left(1-\chi_{K}\right)$ in the $\xi$ direction is comparable to $2^{\frac{d}{2}}$, since on one hand we localize $\left\langle\tau-|\xi|^{2}\right\rangle$ around $2^{d}$, and on the other hand we localize in a region where $\left.|\tau-| \xi\right|^{2} \mid \geq$ $\frac{|\xi|^{2}}{2^{6}}$. 
Proof of (2.18). We wish to prove the following estimate:

$$
\left\|\frac{\left(1-\chi_{K}\right) w}{\left\langle\tau-|\xi|^{2}\right\rangle}\left(\frac{f^{K}}{w} * \frac{g^{K^{c}}}{w}\right)\right\|_{Y^{s}} \lesssim\left\|f^{K}\right\|_{\hat{X}^{s, \frac{1}{2}, 1}}\left\|g^{K^{c}}\right\|_{Y^{s}} .
$$

Since $w=1$ in $K$ we drop $w$ from the ratio $f^{K} / w$. We split the proof into two steps.

Step 1. Estimate in $\langle\xi\rangle^{s} L_{\xi}^{2} L_{\tau}^{1}$.

In this proof we use the $\hat{X}^{s, \frac{1}{2}, 1}$ structure for $f^{K}$ via the inclusion $X^{s, \frac{1}{2}, 1} \subset$ $\langle\xi\rangle^{s} L_{\xi}^{2} L_{\tau}^{1}$. We decompose

$$
\begin{aligned}
& \left(1-\chi_{K}\right)\left(f^{K} * \frac{g^{K^{c}}}{w}\right)=\sum_{k} \sum_{i} \sum_{j} \chi_{K^{c}}\left(f_{i}^{K} * \frac{g_{j}^{K^{c}}}{w}\right)_{k} \\
& =\sum_{i \leq j} \sum_{k=j-10}^{j+2} \chi_{K^{c}}\left(f_{i}^{K} * \frac{g_{j}^{K^{c}}}{w}\right)_{k}+\text { symmetric term } \\
& +\sum_{i} \sum_{j} \sum_{k \leq \text { max }(i, j)-10} \chi_{K^{c}}\left(f_{i}^{K} * \frac{g_{j}^{K^{c}}}{w}\right)_{k} \\
& =J_{1}+\text { symmetric term }+J_{2} .
\end{aligned}
$$

When estimating $J_{1}$ we drop all the weights and use (6.1) to obtain

$$
\begin{aligned}
& \left\|\frac{w J_{1}}{\left\langle\tau-|\xi|^{2}\right\rangle}\right\|_{\langle\xi\rangle^{s} L_{\xi}^{2} L_{\tau}^{1}}^{2} \lesssim \sum_{j} \sum_{k=j-10}^{j+2} 2^{2 k s} 2^{-4 k}\left\|\sum_{i=0}^{j} \chi_{K^{c}}\left(f_{i}^{K} * g_{j}^{K^{c}}\right)_{k}\right\|_{L_{\xi}^{2} L_{\tau}^{1}}^{2} \\
& \lesssim \sum_{j} \sum_{k=j-10}^{j+2} 2^{-4 k} 2^{2(k-j) s}\left(\sum_{i=0}^{j} 2^{i(1-s)}\left\|f_{i}^{K}\right\|_{\langle\xi\rangle^{s} L_{\xi}^{2} L_{\tau}^{1}}\right)^{2}\left\|g_{j}^{K^{c}}\right\|_{\langle\xi\rangle^{s} L_{\xi}^{2} L_{\tau}^{1}}^{2} \\
& \lesssim \sum_{j} \sum_{k=j-10}^{j+2}\left(\sum_{i=0}^{j} 2^{i(1-s)-2 j}\left\|f_{i}^{K}\right\|_{\langle\xi\rangle^{s} L_{\xi}^{2} L_{\tau}^{1}}\right)^{2}\left\|g_{j}^{K^{c}}\right\|_{\langle\xi\rangle^{s} L_{\xi}^{2} L_{\tau}^{1}}^{2} \\
& \lesssim \sum_{j} \sum_{k=j-10}^{j+2}\left\|f^{K}\right\|_{\langle\xi\rangle^{s} L_{\xi}^{2} L_{\tau}^{1}}^{2}\left\|g_{j}^{K^{c}}\right\|_{\langle\xi\rangle^{s} L_{\xi}^{2} L_{\tau}^{1}}^{2} \lesssim\left\|f^{K}\right\|_{\langle\xi\rangle^{s} L_{\xi}^{2} L_{\tau}^{1}}\left\|g^{K^{c}}\right\|_{\langle\xi\rangle^{s} L_{\xi}^{2} L_{\tau}^{1}} \cdot
\end{aligned}
$$

For $J_{2}$, we notice that in order to consider only the nontrivial terms, we must impose the condition $|i-j| \leq 3$. We further split

$$
\begin{aligned}
J_{2} & =\sum_{|i-j| \leq 3} \sum_{k \leq \max (i, j)-10} \sum_{d \geq 2} \chi_{\max (i, j)-10} \chi_{K^{c}}\left(f_{i}^{K} * \frac{g_{j}^{K^{c}}}{w}\right)_{k, d} \\
& +\sum_{|i-j| \leq 3} \sum_{k \leq \max (i, j)-10} \chi_{d \leq 2 \max (i, j)-10} \chi_{K^{c}}\left(f_{i}^{K} * \frac{g_{j}^{K^{c}}}{w}\right)_{k, d} \\
& =J_{21}+J_{22} .
\end{aligned}
$$


The term $J_{21}$ is estimated using (6.2):

$$
\begin{aligned}
& \left\|\frac{w J_{21}}{\left\langle\tau-|\xi|^{2}\right\rangle}\right\|_{\langle\xi\rangle^{s} L_{\xi}^{2} L_{\tau}^{1}} \lesssim \sum_{|i-j| \leq 3} \sum_{k \leq \max (i, j)-10} 2^{k s} 2^{-2 j}\left\|\chi_{K^{c}}\left(f_{i}^{K} * g_{j}^{K^{c}}\right)_{k}\right\|_{\langle\xi\rangle^{s} L_{\xi}^{2} L_{\tau}^{1}} \\
& \lesssim \sum_{|i-j| \leq 3} \sum_{k \leq \max (i, j)-10} 2^{(s+1) k} 2^{-2(s+1) j}\left\|f_{i}^{K}\right\|_{\langle\xi\rangle^{s} L_{\xi}^{2} L_{\tau}}\left\|g_{j}^{K^{c}}\right\|_{\langle\xi\rangle^{s} L_{\xi}^{2} L_{\tau}^{1}} \\
& \lesssim \sum_{|i-j| \leq 3}\left\|f_{i}^{K}\right\|_{\langle\xi\rangle^{s} L_{\xi}^{2} L_{\tau}}\left\|g_{j}^{K^{c}}\right\|_{\langle\xi\rangle^{s} L_{\xi}^{2} L_{\tau}^{1}} \lesssim\left\|f^{K}\right\|_{\langle\xi\rangle^{s} L_{\xi}^{2} L_{\tau}^{1}}\left\|g^{K^{c}}\right\|_{\langle\xi\rangle^{s} L_{\xi}^{2} L_{\tau}^{1}} .
\end{aligned}
$$

The term $J_{22}$ is bounded using (6.3), and keeping the weights,

$$
\begin{aligned}
& \left\|\frac{w}{\left\langle\tau-|\xi|^{2}\right\rangle} \sum_{|i-j| \leq 3} \sum_{k \leq \max (i, j)-10} \sum_{d \leq 2 \max (i, j)-10} \chi_{K^{c}}\left(f_{i}^{K} * \frac{g_{j}^{K^{c}}}{w}\right)_{k, d}\right\|_{\langle\xi\rangle^{s} L_{\xi}^{2} L_{\tau}^{1}} \\
& \lesssim \sum_{|i-j| \leq 3} \sum_{k \leq \max (i, j)-10} \sum_{d \leq 2} 2_{\max (i, j)-10}^{k s} 2^{9 d}\left\|\left(f_{i}^{K} * \frac{g_{j}^{K^{c}}}{w}\right)_{k, d}\right\|_{L_{\xi}^{2} L_{\tau}^{1}} \\
& \lesssim \sum_{|i-j| \leq 3} \sum_{k \leq \max (i, j)-10} \sum_{d \leq 2 \max (i, j)-10} 2^{(s+1) k} 2^{9 d} 2^{-2 j s} 2^{-20 j} \\
& \times\left\|f_{i}^{K}\right\|_{\langle\xi\rangle^{s} L_{\xi}^{2} L_{\tau}^{1}}\left\|g_{j}^{K^{c}}\right\|_{\langle\xi\rangle^{s} L_{\xi}^{2} L_{\tau}^{1}} \\
& \lesssim \sum_{|i-j| \leq 3} \sum_{k \leq \max (i, j)-10} 2^{(s+1) k} 2^{-2(s+1) j}\left\|f_{i}^{K}\right\|\left\|_{\langle\xi\rangle^{s} L_{\xi}^{2} L_{\tau}^{1}}\right\| g_{j}^{K^{c}} \|_{\langle\xi\rangle^{s} L_{\xi}^{2} L_{\tau}^{1}} \\
& \lesssim \sum_{|i-j| \leq 3}\left\|f_{i}^{K}\right\|_{\langle\xi\rangle^{s} L_{\xi}^{2} L_{\tau}^{1}}\left\|g_{j}^{K^{c}}\right\|_{\langle\xi\rangle^{s} L_{\xi}^{2} L_{\tau}^{1}} \lesssim\left\|f^{K}\right\|_{\langle\xi\rangle^{s} L_{\xi}^{2} L_{\tau}^{1}}\left\|g^{K^{c}}\right\|_{\langle\xi\rangle^{s} L_{\xi}^{2} L_{\tau}^{1}} .
\end{aligned}
$$

Combining the estimates for $J_{1}, J_{21}$ and $J_{22}$ we obtain the claim in (2.17).

Step 2. Estimate in $\langle(\xi, \tau)\rangle^{s+1} L^{2}$.

We decompose

$$
\begin{aligned}
& \left(1-\chi_{K}\right)\left(f^{K} * \frac{g^{K^{c}}}{w}\right)=\sum_{d_{3}} \sum_{i} \chi_{K^{c}}\left(f_{i}^{K} * \frac{g^{K^{c}}}{w}\right)_{d_{3}} \\
& =\sum_{i} \sum_{d_{3} \geq 2 i-10} \chi_{K^{c}}\left(f_{i}^{K} * \frac{g^{K^{c}}}{w}\right)_{d_{3}}+\sum_{i} \sum_{d_{3} \leq 2 i-10} \chi_{K^{c}}\left(f_{i}^{K} * \frac{g^{K^{c}}}{w}\right)_{d_{3}} \\
& =L_{1}+L_{2} .
\end{aligned}
$$

We estimate $L_{1}$ by using (6.4) and dropping all the weights:

$$
\begin{aligned}
& \left\|\frac{w L_{1}}{\left\langle\tau-|\xi|^{2}\right\rangle}\right\|_{\langle(\xi, \tau)\rangle^{1+s} L^{2}} \lesssim \sum_{i} \sum_{j} \sum_{d_{3} \geq 2 i-10} 2^{s d_{3}}\left\|\left(f_{i}^{K} * g_{j}^{K^{c}}\right)_{d_{3}}\right\|_{L^{2}} \\
& \lesssim \sum_{i} \sum_{j} 2^{s i} 2^{\min (i, j)} 2^{-(1+s) j} \sum_{d_{3} \geq 2 i-10} 2^{s\left(d_{3}-2 i\right)}\left\|f_{i}^{K}\right\|_{\langle\xi\rangle^{s} L_{\xi}^{2} L_{\tau}}\left\|g_{j}^{K^{c}}\right\|_{\langle(\xi, \tau)\rangle^{1+s} L^{2}} \\
& \lesssim \sum_{i \leq j} 2^{(s+1)(i-j)}\left\|f_{i}^{K}\right\|_{\langle\xi\rangle^{s} L_{\xi}^{2} L_{\tau}^{1}}\left\|g_{j}^{K^{c}}\right\|_{\langle(\xi, \tau)\rangle^{1+s} L^{2}} \\
& +\sum_{i \geq j} 2^{s(i-j)}\left\|f_{i}^{K}\right\|_{\langle\xi\rangle^{s} L_{\xi}^{2} L_{\tau}^{1}}\left\|g_{j}^{K^{c}}\right\|_{\langle(\xi, \tau)\rangle^{1+s} L^{2}} \\
& \lesssim\left\|f^{K}\right\|_{\langle\xi\rangle^{s} L_{\xi}^{2} L_{\tau}^{1}}\left\|g^{K^{c}}\right\|_{\langle(\xi, \tau)\rangle^{1+s} L^{2}} .
\end{aligned}
$$


In order to estimate $L_{2}$ we use (6.5) and the fact that $g$ is supported where $\tau \leq-2^{2 i-5}$. Hence,

$$
\begin{aligned}
& \left\|\frac{w\left(1-\chi_{K}\right)}{\left\langle\tau-|\xi|^{2}\right\rangle} \sum_{i} \sum_{d_{3} \leq 2 i-10}\left(f_{i}^{K} * \frac{g}{w}\right)_{d_{3}}\right\|_{\langle(\xi, \tau)\rangle^{1+s} L^{2}}^{2} \\
& \lesssim\left\|\frac{w\left(1-\chi_{K}\right)}{\left\langle\tau-|\xi|^{2}\right\rangle} \sum_{d_{3}} \sum_{i \geq \frac{d_{3}+10}{2}}\left(f_{i}^{K} * \frac{g}{w}\right)_{d_{3}}\right\|_{\langle(\xi, \tau)\rangle^{1+s} L^{2}}^{2} \\
& \lesssim \sum_{d_{3}} \sum_{i \geq \frac{d_{3}+10}{2}} 2^{(s+19) d_{3}}\left\|\chi_{K^{c}}\left(f_{i}^{K} * \frac{g}{w}\right)_{d_{3}}\right\|_{L^{2}}^{2} \\
& \lesssim \sum_{d_{3}} \sum_{i \geq \frac{d_{3}+10}{2}} 2^{-2 i s} 2^{(s+19) d_{3}} 2^{d_{3}} 2^{-40 i} 2^{-2(s+1) i}\left\|f_{i}\right\|_{\langle\xi\rangle^{s} L_{\xi}^{2} L_{\tau}^{1}}^{2}\|g\|_{\langle(\xi, \tau)\rangle^{1+s} L^{2}}^{2} \\
& \lesssim \sum_{d_{3}} \sum_{i \geq \frac{d_{3}+10}{2}} 2^{(s+20)\left(d_{3}-2 i\right)} 2^{-2 i(s+1)}\left\|f_{i}\right\|_{\langle\xi\rangle^{s} L_{\xi}^{2} L_{\tau}}^{2}\|g\|_{\langle(\xi, \tau)\rangle^{1+s} L^{2}}^{2} \\
& \lesssim\|f\|_{\langle\xi\rangle^{s} L_{\xi}^{2} L_{\tau}^{1}}^{2}\|g\|_{\langle(\xi, \tau)\rangle^{1+s} L^{2}}^{2},
\end{aligned}
$$

which concludes our proof.

\section{ACKNOWLEDGMENTS}

The authors would like to thank James Colliander for encouragement with this project.

\section{REFERENCES}

[1] I. Bejenaru, T. Tao, Sharp well-posedness and ill-posedness results for a quadratic nonlinear Schrödinger equation, J. of Func. Anal. vol. 233, issue 1, pp. 228-259. MR2204680 (2007i:35216)

[2] I. Bejenaru, Quadratic Nonlinear Derivative Schrödinger Equations - Part 2, preprint available on arxiv.

[3] B. Birnir, C. Kenig, G. Ponce, N. Svanstedt, L. Vega, On the ill-posedness of the IVP for the generalized Korteweg-de Vries and nonlinear Schrödinger equations, J. London Math. Soc. (2) 53 (1996), 551-559. MR1396718 (97d:35233)

[4] J. Bourgain, Fourier restriction phenomena for certain lattice subsets and applications to nonlinear evolution equations, Part I, Geometric and Funct. Anal. 3 (1993), 107-156. MR1209299 (95d:35160a)

[5] T. Cazenave, F.B. Weissler, The Cauchy problem for the critical nonlinear Schrödinger equation, Non. Anal. TMA 14 (1990), 807-836. MR1055532(91j:35252)

[6] J. Colliander, J. Delort, C. Kenig, G. Staffilani, Bilinear Estimates and Applications to 2D NLS, Trans. Amer. Math. Soc. 353 (2001), no. 8, 3307-3325. MR1828607(2002d:35186)

[7] M. Christ, J. Colliander, T. Tao, Low-regularity ill-posedness for nonlinear Schrödinger and wave equations, preprint.

[8] C. Kenig, G. Ponce, L. Vega, Quadratic forms for the 1-D semilinear Schrödinger equation, Trans. Amer. Math. Soc. 346 (1996), 3323-3353. MR1357398 (96j:35233)

[9] C. Kenig, G. Ponce, L. Vega, On the ill-posedness of some canonical dispersive equations, Duke Math. J. 106 (2001), 617-633. MR1813239 (2002c:35265)

[10] T. Muramatu, S. Taoka, The initial value problem for the 1-D semilinear Schrödinger equation in Besov spaces, J. Math. Soc. Japan 56 (2004), no. 3, 853-888. MR2071676 (2005h:35325)

[11] K. Nakanishi, H. Takaoka, Y. Tsutsumi, Counterexamples to bilinear estimates related with the KdV equation and the nonlinear Schrödinger equation, Methods and Applications of Analysis 8 (2001), 569-578. MR 1944182 (2004c:35364) 
[12] D. Tataru, Local and global results for wave maps I, Comm. PDE 23 (1998), 1781-1793. MR.1641721 (99j:58209)

[13] T. Tao, Multilinear weighted convolution of $L^{2}$ functions, and applications to nonlinear dispersive equations, Amer. J. Math. 123 (2001), no. 5, 839-908. MR1854113 (2002k:35283)

[14] Y. Tsutsumi, $L^{2}$ solutions for nonlinear Schrodinger equations and nonlinear groups, Funk. Ekva. 30 (1987), 115-125. MR915266 (89c:35143)

Department of Mathematics, University of California, Los Angeles, California 90095

Current address: Department of Mathematics, Texas A \& M University, College Station, Texas 77843

Department of Mathematics, Johns Hopkins University, 3400 N. Charles Street, BalTIMORE, MARYLAND 21218

Current address: Department of Mathematics, Columbia University, 2990 Broadway, New York, New York 10027 